QUARTERLY OF APPLIED MATHEMATICS

VOLUME LXVI, NUMBER 3

SEPTEMBER 2008, PAGES 565-594

S 0033-569X(08)01123-8

Article electronically published on June 5, 2008

\title{
TURBULENT RIVERS
}

\author{
BY \\ BJÖRN BIRNIR \\ Center for Complex and Nonlinear Science, and Department of Mathematics, \\ University of California, Santa Barbara
}

\begin{abstract}
The existence of solutions describing the turbulent flow in rivers is proven. The existence of an associated invariant measure describing the statistical properties of this one-dimensional turbulence is established. The turbulent solutions are not smooth but Hölder continuous with exponent $3 / 4$. The scaling of the solutions' second structure (or width) function gives rise to Hack's law (1957), stating that the length of the main river, in mature river basins, scales with the area of the basin $l \sim A^{h}, h=0.568$ being Hack's exponent.
\end{abstract}

1. Introduction. The flow of water in streams and rivers is a fascinating problem with many applications that has intrigued scientists and laymen for many centuries; see Levi 21. Surprisingly it is still not completely understood even in one or two-dimensional approximations of the full three-dimensional flow. Erosion by water seems to determine the features of the surface of the earth, up to very large scales where the influence of earthquakes and tectonics is felt; see [33, 34, 32, 6, 4, 36. Thus water flow and the subsequent erosion give rise to the various scaling laws known for river networks and river basins; see 12, 8, 9, 10, 11.

One of the best known scaling laws of river basins is Hack's law [16, which states that the area of the basin scales with the length of the main river to an exponent known as Hack's exponent. Careful studies of Hack's exponent, see [11, show that it actually has three ranges, depending on the age and size of the basin, apart from very small and very large scales where it is close to one. The first range corresponds to a spatial roughness coefficient of one half for small channelizing (very young) landsurfaces. This has been explained, see 4 and 13 , as Brownian motion of water and sediment over the channelizing surface. The second range with a roughness coefficient of $2 / 3$ corresponds to the evolution of a young surface forming a convex (geomorphically concave) surface, with young rivers that evolve by shock formation in the water flow. These shocks are called bores (in front) and hydraulic jumps (in rear); see Welsh, Birnir and Bertozzi [36]. Between them sediment is deposited. Finally there is a third range with a roughness

Received May 15, 2007.

2000 Mathematics Subject Classification. Primary 35Q30, 35R60, 60H15, 76D06.

E-mail address: birnir@math.ucsb.edu

(C)2008 Brown University 
coefficient 3/4. This range, which is the largest by far and is associated with what is called the mature landscape, or simply the landscape because it persists for a long time, is what this paper is about. This range is associated with turbulent flow in rivers, and we will develop the statistical theory of turbulent flow in rivers that leads to Hack's exponent.

Starting with the three basic assumptions on river networks: that their structure is self-similar, that the individual streams are self-affine, and that the drainage density is uniform, see 8, river networks possess several scaling laws that are well documented; see 31. These are self-affinity of single channels, which we will call the meandering law, Hack's law, Horton's laws [17. and their refinement Tokunaga's law, the law for the scaling of the probability of exceedance for basin areas and stream lengths, and Langbein's law. The first two laws are expressed in terms of the meandering exponent $m$, or fractal dimension of a river, and Hack's exponent $h$. Horton's laws are expressed in terms of Horton's ratios of link numbers and link lengths in a Strahler ordered river network, Tokunaga's law is expressed in term of the Tokunaga's ratios, the probability of exceedance is expressed by decay exponents, and Langbein's law is given by Langbein's exponents, 8.

In a series of papers, Dodds and Rothman [12, 8, 9, 10, 11, showed that all the above ratios and exponents are determined by $m$ and $h$, the meandering and Hack's exponents; see [16, 12]. The origin of the meandering exponent $m$ is still a mystery, but in this paper we show how Hack's exponent is determined by the scaling exponent of turbulent one-dimensional flow. Specifically, it is determined by the scaling exponent of the second structure function, see [14, in the statistical theory of the turbulent flow.

Two dimensionless numbers, the Reynolds number and the Froude number, are used to characterize turbulent flow in rivers and streams. If we model the river as an open channel with $x$ parameterizing the downstream direction, $y$ the horizontal depth and $U$ is the mean velocity in the downstream direction, then the Reynolds number

$$
R=\frac{f_{\text {turbulent }}}{f_{\text {viscous }}}=\frac{U y}{\nu}
$$

is the ratio of the turbulent and viscous forces whereas the Froude number

$$
F=\frac{f_{\text {turbulent }}}{f_{\text {gravitational }}}=\frac{U}{(g y)^{1 / 2}}
$$

is the ratio of the turbulent and gravitational forces. $\nu$ is the viscosity and $g$ is the gravitational acceleration. Other forces such as surface tension, the centrifugal force and the Coriolis force are insignificant in streams and rivers.

The Reynolds number indicates whether the flow is laminar or turbulent with the transition to turbulence starting at $R=500$ and the flow usually being fully turbulent at $R=2000$. The Froude number measures whether gravity waves, with speed $c=(g y)^{1 / 2}$ in shallow water, caused by some disturbance in the flow, can overcome the flow velocity and travel upstream. Such flows are called tranquil flows, $c>U$, in distinction to rapid or shooting flows, $c<U$, where this cannot happen; they correspond to the Froude numbers

(1) $F<1$, subcritical, $c>U$, 
(2) $F=1$, critical, $c=U$,

(3) $F>1$, supercritical, $c<U$.

Now for streams and rivers the Reynolds number is typically large, $O=10^{5}-10^{6}$, whereas the Froude number is small, typically $O=10^{-1}-10^{-2}$; see [19]. Thus the flows are highly turbulent and ought to be tranquil, but this is not the whole story as we will now explain.

In practice streams and rivers have varied boundaries which are topologically equivalent to a half-pipe. These boundaries are rough and resist the flow and this has led to formulas involving channel resistance. The most popular of these are Chézy's law,

$$
V=u_{c} C r^{1 / 2} s_{o}^{1 / 2}, \quad u_{c}=0.552 \mathrm{~m} / \mathrm{s},
$$

and Manning's law,

$$
V=u_{m} \frac{1}{n} r^{2 / 3} s_{o}^{1 / 2}, \quad u_{m}=1.0 m / s,
$$

where $s_{o}$ is the slope of the channel and $r$ is the hydraulic radius. $C$ is called Chézy's constant and measures inverse channel resistance. $n$ is Manning's roughness coefficient; see [19. We get new effective Reynolds and Froude numbers with these new averaged velocities $V$,

$$
R^{*}=\frac{g}{3 u_{c}^{2} C^{2}} R, \quad F^{*}=\left(\frac{g}{u_{c}^{2} C^{2} s_{o}}\right)^{1 / 2} F .
$$

It turns out that in real rivers the effective Froude number is approximately one and the effective Reynolds number is also one; when $R=500$ for typical channel roughness, $C=73.3$. Thus the transition to turbulence typically occurs in rivers when the effective turbulent forces are equal to the viscous forces.

In this paper we will ignore the boundaries of the river. The point is that in a straight segment of a reasonably deep and wide river the boundaries do not influence the details of the river current in the center, except as a source of flow disturbances. We will simply assume that these disturbances exist, in the flow in the center of the river and not be concerned with how they got there. For theoretical purposes we will conduct a thought experiment where we start with an unstable uniform flow and then put the disturbances in as small white noise. Then the mathematical problem is to determine the statistical theory of the resulting turbulent flow.

The details of the flow close to the boundary are obviously important and give rise to the Prandtl-von Karman universal velocity distribution law for smooth boundaries and the Chézy's and Manning's roughness coefficients for rough boundaries. However, these properties of the flow at the boundaries are a separate problem that will not be addressed in this paper.

The outline of the paper is as follows. In Section 2 we pose the problem. It turns out to be a stochastic initial value problem. In Section 3 we derive a priori estimates necessary for the existence theory and pose the one-dimensional problem solved in this paper. The existence of unique solutions that are Hölder continuous stochastic processes, with Hölder exponent 3/4, is proven in Section 4. The global existence, based on a useful estimate of these solutions, is derived in Subsection 4.1. The existence of a unique invariant measure on the space $L^{2}\left(T^{1}\right)$, where the solutions reside, is proven in Section 5 , following McKean 
23] and Da Prato and Zabczyk 30]. The scaling of the second structure function $(3 / 2)$ is found in Section [6, and the derivation of Hack's law is recalled from [6] and [4].

2. The initial value problem. Consider the Navier-Stokes equation

$$
\begin{aligned}
w_{t}+w \cdot \nabla w & =\nu \Delta w+\nabla p, \\
w(x, 0) & =w_{o},
\end{aligned}
$$

where $\nu$ is the kinematic viscosity, with the incompressibility conditions

$$
\nabla \cdot w=0
$$

Eliminating the pressure $p$ using (2) gives the equation

$$
w_{t}+w \cdot \nabla w=\nu \Delta w+\nabla\left\{\Delta^{-1}\left[\operatorname{trace}(\nabla w)^{2}\right]\right\} .
$$

We want to consider turbulent flow in the center of a wide and deep river and to do that we consider the flow to be in a box and impose periodic boundary conditions on the box. Since we are mostly interested in what happens in the direction along the river we take our $x$-axis to be in that direction.

We will assume that the river flows fast and pick an initial condition of the form

$$
w(0)=U_{o} e_{1},
$$

where $U_{o}$ is a large constant and $e_{1}$ is a unit vector in the $x$ direction. Clearly this initial condition is not sufficient because the fast flow will be unstable and the white noise, ubiquitous in nature, will grow into small velocity and pressure oscillations; see for example [1]. But we perform a thought experiment where white noise is introduced into the fast flow at $t=0$. This experiment may be hard to perform in nature but it is easily done numerically. This means that we should look for a solution of the form

$$
w(x, t)=U_{o} e_{1}+u(x, t),
$$

where $u(x, t)$ is smaller that $U_{o}$ but not necessarily small. However, in a small initial interval $\left[0, t_{o}\right], u$ is small and satisfies the equation (3) linearized about the fast flow $U_{o}$,

$$
\begin{aligned}
u_{t}+U_{o} \nabla u & =\nu \Delta u+f, \\
u(x, 0) & =0,
\end{aligned}
$$

driven by the noise

$$
f=\sum_{k \neq 0} h_{k}^{1 / 2} d \beta_{t}^{k} e_{k}
$$

The $e_{k}=e^{2 \pi i k \cdot x}$ are (three-dimensional) Fourier components and each comes with its own independent Brownian motion $\beta_{t}^{k}$. None of the coefficients of the vectors $h_{k}^{1 / 2}=$ $\left(h_{1}^{1 / 2}, h_{2}^{1 / 2}, h_{3}^{1 / 2}\right)$ vanishes because the instabilities are seeded by truly white noise (white both is space and in time). $f$ is not white in space because the coefficients $h_{k}^{1 / 2}$ must have some decay in $k$ so that the noise term in (6) makes sense. However to determine the decay of the $h_{k}^{1 / 2} \mathrm{~s}$ will now be part of the problem. The form of the noise expresses the fact that in turbulent flow there is a continuous source of small white noise that grows and saturates into turbulent noise that drives the fluid flow. The decay of the coefficients $h_{k}^{1 / 2}$ expresses the spatial coloring of this larger noise in turbulent flow. 
The justification for considering the initial value problem (6) is that for a short time interval $\left[0, t_{o}\right]$ we can ignore the nonlinear terms

$$
-u \cdot \nabla u+\nabla\left\{\Delta^{-1}\left[\operatorname{trace}(\nabla u)^{2}\right]\right\}
$$

in the equation (3). But this is only true for a short time $t_{o}$; after this time we have to start with the solution of (6),

$$
u_{o}(x, t)=\sum_{k \neq 0} h_{k}^{1 / 2} \int_{0}^{t} e^{-\left(4 \pi^{2} \nu k^{2}+2 \pi i U_{o} k\right)(t-s)} d \beta_{s}^{k} e_{k}(x),
$$

as the first iterate in the integral equation

$$
u(x, t)=u_{o}(x, t)+\int_{t_{o}}^{t} K(t-s) *\left[-u \cdot \nabla u+\nabla \Delta^{-1}\left(\operatorname{trace}(\nabla u)^{2}\right)\right] d s,
$$

where $K$ is the (oscillatory heat) kernal in (7). In other words, to get the turbulent solution we must take the solution of the linear equation (6) and use it as the first term in (8). It will also be the first guess in Picard iteration. The solution of (6) can be written in the form

$$
u_{o}(x, t)=\sum_{k \neq 0} h_{k}^{1 / 2} A_{t}^{k} e_{k}(x)
$$

where the

$$
A_{t}^{k}=\int_{0}^{t} e^{-\left(4 \pi^{2} \nu k^{2}+2 \pi i U_{o} k\right)(t-s)} d \beta_{s}^{k}
$$

are independent Orstein-Uhlenbeck processes with mean zero; see for example [30].

Now it is easy to see that the solution $u(x, t)$ of the integral equation (8) satisfies the driven Navier-Stokes equation

$$
\begin{aligned}
u_{t}+U_{o} \nabla u & =\nu \Delta u-u \cdot \nabla u+\nabla \Delta^{-1}\left(\operatorname{trace}(\nabla u)^{2}\right)+\sum_{k \neq 0} h_{k}^{1 / 2} \frac{d \beta_{t}^{k}}{d t} e_{k}, \\
u(x, 0) & =u^{0}(x),
\end{aligned}
$$

and the above argument is the justification for studying the initial value problem (10). We will do so from here on. The solution $u$ of (10) still satisfies the periodic boundary conditions and the incompressibility condition

$$
\nabla \cdot u=0
$$

The mean of the solution $u_{o}$ of the linear equation (6) is zero by the formula (7), and this implies that the solution $u$ of (10) also has mean zero,

$$
\bar{u}(t)=\int_{\mathbb{T}^{3}} u(x, t) d x=0
$$

if the initial data also has mean zero, $\bar{u}^{0}=0$.

If we take the initial data equal to zero in (10), then the undriven $f=0$ linear problem (6) is stable and one may have to wait a while until the driving in (10) destabilizes the initial conditions $u^{0}=0$, corresponding to the uniform flow solution (6) of the 
Navier-Stokes equation (3). However, in turbulent flow the uniform flow is immediately destabilized due to large fluctuations; see [2 for a discussion of this mechanism.

3. A priori estimates. In this section we will explain the probabilistic setting and prove the a priori estimates necessary for the existence proof. The problem to be solved will also be posed.

We let $(\Omega, \mathcal{F}, P), \Omega$ is a set (of events) and $\mathcal{F}$ a $\sigma$ algebra on $\Omega$, denote a probability space with $P$ the probability measure of Brownian motion and $\mathcal{F}_{t}$ a filtration generated by all the Brownian motions $\beta_{t}^{k}$ on $[t, \infty)$. We denote by $\mathcal{V}[0, T]$ the space having the following properties; see Oksendal [27.

Definition $1 . \mathcal{V}[0, T]$ is the space of functions satisfying the conditions:

(1) $f(\omega, t): \Omega \times \mathbb{R}^{+} \rightarrow \mathbb{R}$ is measurable with respect to $\mathcal{F} \times \mathcal{B}$ where $\mathcal{B}$ is the $\sigma$-algebra of the Borel sets on $[0, \infty), \omega \in \Omega$,

(2) $f(\omega, t)$ is adapted to the filtration $\mathcal{F}_{t}$,

$$
E\left(\int_{0}^{T} f^{2}(\omega, t) d t\right)<\infty .
$$

We denote by $\mathcal{W}[0, T]$ the space of functions, whose $W^{(m, p)}$ Sobolev norm $\|u\|_{(m, p)}(\omega, t)$ lies in $\mathcal{V}[0, T]$, and we let $\mathcal{L}_{(m, p)}^{2}$ denote the space of functions in $W^{(m, p)}$ whose Sobolev norm lies in $L^{2}(\Omega, P)$. The norm in the space $\mathcal{L}_{(m, p)}^{2}$ is

$$
\left[E\left(\|u\|_{(m, p)}^{2}\right)\right]^{1 / 2} \text {. }
$$

In particular for $p=2$, we get the norm

$$
\|u\|_{\mathcal{L}_{(m, 2)}^{2}}^{2}=E\left(\left|\left(1-\Delta^{2}\right)^{m / 2} u\right|_{2}^{2}\right) .
$$

Here $E$ denotes the expectation in $L^{2}(\Omega, P)$ and $|\cdot|_{2}$ denotes the $L^{2}\left(\mathbb{T}^{3}\right)$ norm. We will proved the existence of solutions in the subspace of continuous functions $C\left([0, T] ; \mathcal{L}_{(m, p)}^{2}\right)$ in $\mathcal{W}[0, T]$.

Let $\langle\cdot, \cdot\rangle$ denote the inner product in $L^{2}\left(\mathbb{T}^{3}\right)$. The following a priori estimates provide the foundation of the probabilistic version of Leray's theory.

Lemma 1. The $L^{2}$ norms $|u|_{2}(\omega, t)$ and $|\nabla u|_{2}(\omega, t)$ satisfy the identity

$$
d|u|_{2}^{2}+2 \nu|\nabla u|_{2}^{2} d t=2 \sum_{k \neq 0}\left\langle u, h_{k}^{1 / 2} e_{k}\right\rangle d \beta_{t}^{k}+\sum_{k \neq 0} h_{k} d t
$$

and the bounds

$$
\begin{array}{r}
|u|_{2}^{2}(\omega, t) \leq|u|_{2}^{2}(0) e^{-2 \nu \lambda_{1} t}+2 \sum_{k \neq 0} \int_{0}^{t} e^{-2 \nu \lambda_{1}(t-s)}\left\langle u, h_{k}^{1 / 2} e_{k}\right\rangle d \beta_{s}^{k} \\
+\frac{1-e^{-2 \nu \lambda_{1} t}}{2 \nu \lambda_{1}} \sum_{k \neq 0} h_{k}, \\
\int_{0}^{t}|\nabla u|_{2}^{2}(\omega, s) d s \leq \frac{1}{2 \nu}|u|_{2}^{2}(0)+\frac{1}{\nu} \sum_{k \neq 0} \int_{0}^{t}\left\langle u, h_{k}^{1 / 2} e_{k}\right\rangle d \beta_{s}^{k}+\frac{t}{2 \nu} \sum_{k \neq 0} h_{k},
\end{array}
$$


where $\lambda_{1}$ is the smallest eigenvalue of $-\Delta$ with vanishing boundary conditions on the box $[0,1]^{3}$ and $h_{k}=\left|h_{k}^{1 / 2}\right|^{2}$. The expectations of these norms are also bounded:

$$
\begin{aligned}
E\left(|u|_{2}^{2}\right)(t) & \leq E\left(|u|_{2}^{2}(0)\right) e^{-2 \nu \lambda_{1} t}+\frac{1-e^{-2 \nu \lambda_{1} t}}{2 \nu \lambda_{1}} \sum_{k \neq 0} h_{k}, \\
E\left(\int_{0}^{t}|\nabla u|_{2}^{2}(s) d s\right) & \leq \frac{1}{2 \nu} E\left(|u|_{2}^{2}(0)\right)+\frac{t}{2 \nu} \sum_{k \neq 0} h_{k} .
\end{aligned}
$$

Proof. The identity (13) follows from Leray's theory and Ito's Lemma. We apply Ito's Lemma to the $L^{2}$ norm of $u$ squared,

$$
d \int_{\mathbb{T}^{3}}|u|^{2} d x=2 \int_{\mathbb{T}^{3}} \frac{\partial u}{\partial t} \cdot u d x d t+2 \sum_{k \neq 0} \int_{\mathbb{T}^{3}} u \cdot h_{k}^{1 / 2} e_{k} d x d \beta_{t}^{k}+\sum_{k \neq 0} h_{k} \int_{\mathbb{T}^{3}} d x d t,
$$

where $k \in \mathbb{Z}^{3}$ and $h_{k}^{1 / 2} \in \mathbb{R}^{3}$. Now by use of the Navier-Stokes equation (3),

$$
\begin{aligned}
d|u|^{2}=2 \int_{\mathbb{T}^{3}} \nu \Delta u \cdot u+ & \left(-u \cdot \nabla u+\nabla \Delta^{-1}\left(\operatorname{trace}(\nabla u)^{2}\right) \cdot u d x\right. \\
& +2 \sum_{k \neq 0} \int_{\mathbb{T}^{3}} u \cdot h_{k}^{1 / 2} e_{k} d x d \beta_{t}^{k}+\sum_{k \neq 0} h_{k} d t \\
=-2 \nu|\nabla u|_{2}^{2} d t & +2 \sum_{k \neq 0} \int_{\mathbb{T}^{3}} u \cdot h_{k}^{1 / 2} e_{k} d x d \beta_{t}^{k}+\sum_{k \neq 0} h_{k} d t
\end{aligned}
$$

since the divergent-free vector $u$ is orthogonal both to the gradient $\nabla \Delta^{-1}\left(\operatorname{trace}(\nabla u)^{2}\right)$ and $u \cdot \nabla u$ by the divergence theorem. The first term in the last expression is obtained by integration by parts. This is the identity (13). The inequality (14) is obtained by applying Poincaré's inequality

$$
\lambda_{1}|u|_{2}^{2} \leq|\nabla u|_{2}^{2},
$$

where $\lambda_{1}$ is the smallest eigenvalue of $-\Delta$ with vanishing boundary conditions on the cube $[0,1]^{3}$. By Poincaré's inequality,

$$
\begin{aligned}
d|u|_{2}^{2} & +2 \nu \lambda_{1}|u|_{2}^{2} d t \leq d|u|_{2}^{2}+2 \nu|\nabla u|_{2}^{2} d t \\
& =2 \sum_{k \neq 0}\left\langle u, h_{k}^{1 / 2} e_{k}\right\rangle d \beta_{t}^{k}+\sum_{k \neq 0} h_{k} d t .
\end{aligned}
$$

Solving the inequality gives (14). (15) is obtained by integrating (13),

$$
|u|_{2}^{2}(t)+2 \nu \int_{0}^{t}|\nabla u|_{2}^{2}(s) d s=|u|_{2}^{2}(0)+2 \sum_{k \neq 0} \int_{0}^{t}\left\langle u, h_{k}^{1 / 2} e_{k}\right\rangle d \beta_{s}^{k}+t \sum_{k \neq 0} h_{k}
$$

and dropping $|u|_{2}^{2}(t)>0$.

Finally we take the expectations of (14) and (15) to obtain respectively (16) and (17), using that the function $\left\langle u, h_{k}^{1 / 2} e_{k}\right\rangle(\omega, t)$ is adapted to the filtration $\mathcal{F}_{t}$. 
3.1. The model of river flow. In a deep and wide river it is reasonable to think that the directions transverse to the main flow, $y$ the direction across the river, and $z$ the horizontal direction, play a secondary role in the generation of turbulence. As a first approximation to the flow in the center of a deep and wide, fast-flowing river we will now drop these directions. Of course $y$ and $z$ play a role in the motion of the large eddies in the river, but their motion is relatively slow compared to the smaller scale turbulence. Thus our initial value problem (10) becomes

$$
\begin{aligned}
u_{t}+U_{o} u_{x} & =u_{x x}-u u_{x}+\partial_{x}^{-1}\left(\left(u_{x}\right)^{2}\right)-b+\sum_{k \neq 0} h_{k}^{1 / 2} \frac{d \beta_{t}^{k}}{d t} e_{k}, \\
u(x, 0) & =u^{0}(x),
\end{aligned}
$$

where the constant

$$
b=\int_{0}^{1} \partial_{x}^{-1}\left(\left(u_{x}\right)^{2}\right) d x
$$

keeps the mean of $u$ equal to zero. The problem we pose is to find a Sobolev space determining the decay of the (turbulent) noise coefficients $h_{k}^{1 / 2}$ so that there exists a unique solution of (20) in this space. This solution will necessarily be a stochastic process and we want to determine the statistical theory associated with this stochastic process.

We still have periodic boundary conditions on the unit interval, but the incompressibility condition can be dropped. Recall from Equation (12) that the mean $\bar{u}$ is still zero. This equation now describes the turbulent flow in the center of a relatively straight section of a fast river. The full three-dimensional pressure term is modeled by the pressure variations in the downstream directions only. This is a model

$$
\nabla p \approx \partial_{x}^{-1}\left(\left(u_{x}\right)^{2}\right)-b
$$

of the pressure gradient in the river flow. The full three-dimensional flow will be treated in a subsequent publication [3].

The one-dimensional version of Lemma 1 is

Corollary 1. In one dimension the $L^{2}$ norms of $u$ and $u_{x}$ satisfy

$$
\begin{array}{r}
|u|_{2}^{2}(\omega, t) \leq|u|_{2}^{2}(0) e^{-2 \nu \lambda_{1} t}+2 \sum_{k \neq 0} \int_{0}^{t} e^{-2 \nu \lambda_{1}(t-s)}\left\langle u, h_{k}^{1 / 2} e_{k}\right\rangle d \beta_{s}^{k} \\
+\frac{1-e^{-2 \nu \lambda_{1} t}}{2 \nu \lambda_{1}} \sum_{k \neq 0} h_{k}, \\
\int_{0}^{t}\left|u_{x}\right|_{2}^{2}(\omega, s) d s \leq \frac{1}{2 \nu}|u|_{2}^{2}(0)+\frac{1}{\nu} \sum_{k \neq 0} \int_{0}^{t}\left\langle u, h_{k}^{1 / 2} e_{k}\right\rangle d \beta_{s}^{k}+\frac{t}{2 \nu} \sum_{k \neq 0} h_{k},
\end{array}
$$


where $\lambda_{1}$ is the smallest eigenvalue of $-\partial_{x}^{2}$ with vanishing boundary conditions on the interval $[0,1]$. The expectations of these norms are also bounded:

$$
\begin{aligned}
E\left(|u|_{2}^{2}\right)(t) & \leq E\left(|u|_{2}^{2}(0)\right) e^{-2 \nu \lambda_{1} t}+\frac{1-e^{-2 \nu \lambda_{1} t}}{2 \nu \lambda_{1}} \sum_{k \neq 0} h_{k}, \\
E\left(\int_{0}^{t}\left|u_{x}\right|_{2}^{2}(s) d s\right) & \leq \frac{1}{2 \nu} E\left(|u|_{2}^{2}(0)\right)+\frac{t}{2 \nu} \sum_{k \neq 0} h_{k} .
\end{aligned}
$$

4. Existence of turbulent solutions. In this section we prove the existence of the turbulent solutions of the initial value problem (20). The following theorem states the existence of turbulent solutions in one dimension. First we write the initial value problem (20) as an integral equation,

$$
\left.u(x, t)=u_{o}(x, t)+K(t) * u^{0}+\int_{0}^{t} K(t-s) *\left[-\frac{1}{2}\left(u^{2}\right)_{x}+\partial_{x}^{-1}\left(u_{x}\right)^{2}\right)-b\right] d s .
$$

Here $K$ is the oscillatory heat kernal (7) in one dimension and

$$
u_{o}(x, t)=\sum_{k \neq 0} h_{k}^{1 / 2} A_{t}^{k} e_{k}(x)
$$

the $A_{t}^{k} \mathrm{~s}$ being the Orstein-Uhlenbeck processes from Equation (9).

4.1. An estimate of the turbulent solution. The mechanism of the turbulence production is fast oscillations driving large turbulent noise, which was initially seeded by small white noise. These fast oscillations are generated by the fast constant flow $U_{o}$ and their velocity increases with $U_{o}$. The larger $U_{o}$ is the more efficient this mechanism becomes. The following lemma plays a key role in the proof of the useful estimate of the turbulent solution. It is a version of the Riemann-Lebesgue lemma, which captures the averaging effect (mixing) of the oscillations.

Lemma 2. Let the Fourier transform in time be

$$
\tilde{w}=\int_{0}^{T} w(s) e^{-2 \pi i k U_{o} s} d s,
$$

where $w$ and $\tilde{w}$ are vectors with three components, periodic with period $T=\frac{n}{k U_{o}}, n \in \mathbb{Z}$. Then

$$
\tilde{w}=\widetilde{\partial w}
$$

where

$$
\partial w=\frac{1}{2}\left(w(s)-w\left(s+\frac{1}{2 k U_{o}}\right)\right)=\frac{1}{2} \int_{s+\frac{1}{2\left|k U_{o}\right|}}^{s} \frac{\partial w}{\partial r} d r
$$

and $\precsim w$ satisfies the estimate

$$
|ð w| \leq \frac{1}{4\left|k U_{o}\right|} \operatorname{ess} \sup _{\left[s, s+\frac{1}{\left.2 k U_{o}\right]}\right]}\left|\frac{\partial w}{\partial s}\right| .
$$


Proof. The proof is similar to the proof of the Riemann-Lebesgue lemma for the Fourier transform in time:

$$
\begin{aligned}
\tilde{w}(k) & =\int_{0}^{T} w(s) e^{-2 \pi i k U_{o} s} d s \\
& =-\int_{0}^{T} w(s) e^{-2 \pi i k U_{o}\left(s-\frac{1}{2 k U_{o}}\right)} d s \\
& =-\int_{0}^{T} w\left(s+\frac{1}{2 k U_{o}}\right) e^{-2 \pi i k U_{o} s} d s .
\end{aligned}
$$

Taking the average of the first and the last expression we get

$$
\tilde{w}=\frac{1}{2} \int_{0}^{T}\left(w(s)-w\left(s+\frac{1}{2 k U_{o}}\right)\right) e^{-2 \pi i k U_{o} s} d s=\widetilde{\partial w} .
$$

Now

$$
\begin{aligned}
|\widetilde{\partial} w| & =\frac{1}{2}\left|\left(w(s)-w\left(s+\frac{1}{2 k U_{o}}\right)\right)\right| \\
& \leq \frac{1}{2} \int_{s}^{s+\frac{1}{2 k U_{o}}}\left|\frac{\partial w}{\partial r}\right| d r \\
& \leq \frac{1}{4\left|k U_{o}\right|} \operatorname{ess} \sup _{\left[s, s+\frac{1}{\left.2 \mid k U_{o}\right]} \mid\right.}\left|\frac{\partial w}{\partial s}\right| .
\end{aligned}
$$

The lemma allows us to estimate the Fourier transform (in $t$ ) of $w$ in terms of the time derivative of $w$, with a gain of $\left(k U_{o}\right)^{-1}$. The next lemma gives a similar estimate with $w$ multiplied by the oscillatory exponential.

LEMMA 3.

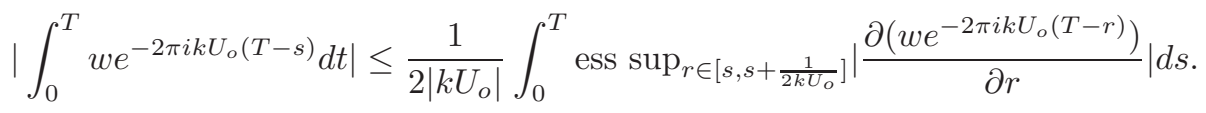

Proof. We take the complex conjugate of the equality (27) in Lemma 2 and multiply by $e^{-2 \pi i k U_{o} T}$ to get the equality

$$
\int_{0}^{T} w e^{-2 \pi i k U_{o}(T-s)} d s=\frac{1}{2} \int_{0}^{T} \int_{s+\frac{1}{2 k U_{o}}}^{s} \frac{\partial w}{\partial r} d r e^{-2 \pi i k U_{o}(T-s)} d s .
$$

This is equivalent to the equality

$$
\begin{array}{r}
\int_{0}^{T}\left(w+\pi i k U_{o} \int_{s+\frac{1}{2 k U_{o}}}^{s} \frac{\partial w}{\partial r} d r\right) e^{-2 \pi i k U_{o}(T-s)} d s \\
=\frac{1}{2} \int_{0}^{T} \int_{s+\frac{1}{2 k U_{o}}}^{s} \frac{\partial w e^{-2 \pi i k U_{o}(T-r)}}{\partial r} e^{2 \pi i k U_{o}(T-r)} d r e^{-2 \pi i k U_{o}(T-s)} d s .
\end{array}
$$

Now

$$
\begin{aligned}
\int_{s+\frac{1}{2 k U_{o}}}^{s} \frac{\partial w}{\partial r} d r e^{-2 \pi i k U_{o}(T-s)} & =w(s) e^{-2 \pi i k U_{o}(T-s)}+w\left(s+\frac{1}{2 k U_{o}}\right) e^{-2 \pi i k U_{o}\left(T-\left(s+\frac{1}{2 k U_{o}}\right)\right)} \\
& =2 w(s) e^{-2 \pi i k U_{o}(T-s)}+\int_{s+\frac{1}{2 k U_{o}}}^{s} \frac{\partial w(r) e^{-2 \pi i k U_{o}(T-r)}}{\partial r} d r .
\end{aligned}
$$


Substituting into the formula above we obtain the identity

$$
\begin{aligned}
(1 & \left.+2 \pi i k U_{o}\right) \int_{0}^{T} w e^{-2 \pi i k U_{o}(T-s)} d s \\
& =\frac{1}{2} \int_{0}^{T} \int_{s+\frac{1}{2 k U_{o}}}^{s} \frac{\partial w e^{-2 \pi i k U_{o}(T-r)}}{\partial r}\left(2 \pi i k U_{o}+e^{2 \pi i k U_{o}(s-r)}\right) d r d s .
\end{aligned}
$$

Then taking the absolute value and applying the triangle inequality we get

$$
\begin{aligned}
(1 & \left.+4 \pi^{2} k^{2} U_{o}^{2}\right)^{\frac{1}{2}}\left|\int_{0}^{T} w e^{-2 \pi i k U_{o}(T-s)} d t\right| \\
& \leq \frac{\left(1+2 \pi\left|k U_{o}\right|\right)}{4\left|k U_{o}\right|} \int_{0}^{T} \operatorname{ess} \sup _{r \in\left[s, s+\frac{1}{2 k U_{o}}\right]}\left|\frac{\partial\left(w e^{-2 \pi i k U_{o}(T-r)}\right)}{\partial r}\right| d s .
\end{aligned}
$$

Dividing the last inequality by $\left(1+4 \pi^{2} k^{2} U_{o}^{2}\right)^{\frac{1}{2}}$ and using the inequality $(a+b)^{2} \leq$ $2\left(a^{2}+b^{2}\right)$ gives the result.

We will also need the following technical lemma.

Lemma 4. The integral

$$
\int_{0}^{t}(2 \pi|k|)^{p} e^{-\left(4 \pi^{2} \nu k^{2}+2 \pi i k U_{o}\right)(t-s)} d s
$$

is bounded by

$$
(2 \pi)^{p} \int_{0}^{t}|k|^{p} e^{-4 \pi^{2} \nu k^{2}(t-s)} d s \leq C t^{1-\frac{p}{2}}
$$

for $0 \leq p<2$, where $C$ is a constant. In particular,

$$
\int_{t-\delta}^{t}(2 \pi|k|)^{p} e^{-\left(4 \pi^{2} \nu k^{2}+2 \pi i k U_{o}\right)(t-s)} d s \leq C \delta^{1-\frac{p}{2}}
$$

and

$$
\int_{0}^{t-\delta}(2 \pi|k|)^{p} e^{-\left(4 \pi^{2} \nu k^{2}+2 \pi i k U_{o}\right)(t-s)} d s \leq C \delta^{1-\frac{p}{2}} .
$$

Proof. We estimate the integral

$$
\begin{array}{r}
\int_{0}^{t}|k|^{p} e^{-4 \pi^{2} \nu k^{2}(t-s)} d s=\int_{0}^{t}|k|^{p} e^{-4 \pi^{2} \nu k^{2} r} d r \\
\leq\left(\frac{p}{8 \pi^{2} \nu}\right)^{\frac{p}{2}} e^{-p} \int_{0}^{t} r^{-\frac{p}{2}} d r=C t^{1-\frac{p}{2}}
\end{array}
$$

where

$$
k=\frac{1}{2 \pi} \sqrt{\frac{p}{2 \nu r}}
$$

is the value of $k$ where the integrand achieves its maximum. The second estimate (32) is obtained by first integrating and then computing the maximum of $|k|^{p} e^{-4 \pi^{2} \nu k^{2} \delta}$.

If $\frac{q}{p}$ is a rational number, let $\frac{q}{p}^{+}$denote any real number $s>\frac{q}{p}$. 
Theorem 1. If the velocity $U_{o}$ of the uniform flow is sufficiently large and the initial function $u_{o}(x, t)$ and the initial condition $u^{0}(x)$ in the integral equation (26) satisfy

$$
\begin{aligned}
& E\left(\left\|u_{o}\right\|_{\frac{5}{4}}^{2}\right)+E\left(\left\|K * u^{0}\right\|_{\frac{5}{4}}^{2}\right) \\
& =\frac{1}{2} \sum_{k \neq 0} \frac{\left(1+(2 \pi|k|)^{(5 / 2)^{+}}\right)}{(2 \pi|k|)^{2}} h_{k}+E\left(\left\|K * u^{0}\right\|_{\frac{5}{4}}^{2}\right) \\
& \quad \leq \frac{1}{24} C\left|U_{o}\right|-\frac{1}{16},
\end{aligned}
$$

then the solution of the integral equation (26) is uniformly bounded in $\mathcal{L}_{\left(\frac{5}{4}+, 2\right)}^{2}$. Namely,

$$
\operatorname{ess} \sup _{t \in[0, \infty)} E\left(\|u\|_{\frac{5}{4}^{+}}^{2}\right)(t)<C\left|U_{o}\right|
$$

where $C$ is a constant and $K$ is the oscillating heat kernel from (7).

Corollary 2 (Onsager's Conjecture). The solutions of the integral equation (26) are Hölder continuous with exponent $3 / 4$.

REMARK 1. The hypothesis (33) is the answer to the question we posed in Section 2 and Subsection 3.1. namely how fast the coefficients $h_{k}^{1 / 2}$ had to decay in Fourier space. They have to decay sufficiently fast for the expectation of the $H^{\frac{5}{4}}{ }^{+}=W^{\left(\frac{5}{4}+, 2\right)}$ Sobolev norm of the initial function $u_{o}$ to be finite. In other words the $\mathcal{L}_{\left(\frac{5}{4}+2\right)}^{2}$ norm of the initial function $u_{o}$ has to be finite.

We now prove the theorem with the help of one more lemma in addition to those above.

Proof. We write the integral equation (26) in the form

$$
\left.u(x, t)=\sum_{k \neq 0}\left[h_{k}^{1 / 2} A_{t}^{k}+\frac{1}{2} \int_{0}^{t} e^{-\left(4 \pi^{2} \nu k^{2}+2 \pi i k U_{o}\right)(t-s)}\left(2 \widehat{\partial_{x}^{-1}\left(u_{x}\right.}\right)^{2}-\widehat{\partial_{x} u^{2}}\right)(k, s) d s\right] e_{k}(x),
$$

where $e_{k}=e^{2 \pi i k x}$ are the Fourier components and the $A_{t}^{k}$ are the Ornstein-Uhlenbeck processes (9). We have used that the constant $b$ in (26) keeps the $k=0$ Fourier coefficient of $u$ equal to zero, and we omit the initial conditions to begin with. They will be added in the last step of the estimates.

By the orthogonality of the $e_{k} \mathrm{~s}$ the $L^{2}$ norm of $u$ is

$$
|u|_{2}^{2}=\sum_{k \neq 0}\left[h_{k}\left|A_{t}^{k}\right|^{2}+\frac{1}{4}\left|\int_{0}^{t} e^{-\left(4 \pi^{2} \nu k^{2}+2 \pi i k U_{o}\right)(t-s)}\left(2 \widehat{\partial_{x}^{-1}\left(u_{x}\right)^{2}}-\widehat{\partial_{x} u^{2}}\right)(k, s) d s\right|^{2}\right] .
$$

We split the $t$ integral into the integral from 0 to $t-\delta$, where $\delta$ is a small number, and the integral from $t-\delta$ to $t$. This is done to first avoid the singularities of the spatial derivatives of the heat kernel at $s=t$ and then to deal with these singularities in the latter integral. First we estimate the integral from $t-\delta$ to $t$. The $L^{2}$ norm of this integral is

$$
\left.\sum_{k \neq 0} \frac{1}{4} \mid \int_{t-\delta}^{t} e^{-\left(4 \pi^{2} \nu k^{2}+2 \pi i k U_{o}\right)(t-s)}\left(2 \widehat{\partial_{x}^{-1}\left(u_{x}\right.}\right)^{2}-\widehat{\partial_{x} u^{2}}\right)\left.(k, s) d s\right|^{2}
$$


and it is estimated by

$$
\begin{aligned}
& \left.\leq\left.\delta^{2} \underset{[t-\delta, t]}{\operatorname{ess} \sup _{1}}\left(\sum_{k \neq 0} 4 \mid \partial_{x}^{-1\left(u_{x}\right.}\right)^{2}\right|_{2} ^{2}(k)+\sum_{k \neq 0}\left|\widehat{u u_{x}}\right|_{2}^{2}(k)\right) \\
& \left.\leq \delta^{2} E \operatorname{ess} \sup 4\left|\partial_{x}^{-1}\left(u_{x}\right)^{2}-b\right|_{2}^{2}+\left|u u_{x}\right|_{2}^{2}\right) \\
& {[t-\delta, t]} \\
& \leq C \delta^{2} \operatorname{ess}_{[t-\delta, t]}^{\sin }\left(\left|u_{x}\right|_{4}^{4}+|u|_{\infty}^{2}\left|u_{x}\right|_{2}^{2}\right) \\
& \leq C \delta^{2} \operatorname{ess~sup}_{[t-\delta, t]}\left(\left|u_{x}\right|_{4}^{4}+\left|u_{x}\right|_{2}^{4}\right)
\end{aligned}
$$

by Plancherel's identity and by the Gagliardo-Nirenberg inequalities,

$$
|u|_{\infty} \leq C\|u\|_{\frac{1}{2}} \leq C\left|u_{x}\right|_{2},
$$

in the space $L^{2}$ with mean zero, where $\delta$ is independent of $U_{o}$ and $C$ is a constant.

The integral from 0 to $t-\delta$,

$$
\sum_{k \neq 0} \frac{1}{4}\left|\int_{0}^{t-\delta} e^{-\left(4 \pi^{2} \nu k^{2}+2 \pi i k U_{o}\right)(t-s)}\left(2 \widehat{\partial_{x}^{-1}\left(u_{x}\right)^{2}}-\widehat{\partial_{x} u^{2}}\right)(k, s) d s\right|^{2},
$$

is estimated by use of Lemmas 2 , 3 and 4 . First consider

$$
\begin{aligned}
& \sum_{k \neq 0}\left|\int_{0}^{t-\delta} e^{-\left(4 \pi^{2} \nu k^{2}+2 \pi i k U_{o}\right)(t-s)} \widehat{\partial_{x} u^{2}}(k, s) d s\right|^{2} \\
= & \sum_{k \neq 0}\left|\int_{0}^{t-\delta} e^{-\left(4 \pi^{2} \nu k^{2}+2 \pi i k U_{o}\right)(t-s)} 2 \pi i k \hat{u} * \hat{u} d s\right|^{2} .
\end{aligned}
$$

We write

$$
\begin{aligned}
e^{-\left(2 \pi i k U_{o}\right)(T-s)} \hat{u} * \hat{u} & =e^{-\left(2 \pi i k U_{o}\right)(T-s)} \sum_{l \neq k, 0} \hat{u}(k-l) \hat{u}(l) \\
& =\sum_{l \neq k, 0} e^{-\left(2 \pi i(k-l) U_{o}\right)(T-s)} \hat{u}(k-l) e^{-\left(2 \pi i l U_{o}\right)(T-s)} \hat{u}(l) \\
& =\hat{u} e^{-\left(2 \pi i k U_{o}\right)(T-s)} * \hat{u} e^{-\left(2 \pi i k U_{o}\right)(T-s)} .
\end{aligned}
$$

Then Fourier transforming the Navier-Stokes equation and multiplying it with an exponential gives

$$
\begin{aligned}
& \frac{\partial\left(e^{-\left(2 \pi i k U_{o}\right)(T-s)} \hat{u}\right)}{\partial s}=-4 \pi^{2} \nu k^{2} \hat{u} e^{-\left(2 \pi i k U_{o}\right)(T-s)} \\
& -\frac{1}{2} \widehat{\partial_{x} u^{2}} e^{-\left(2 \pi i k U_{o}\right)(T-s)}+\partial_{x} \widehat{-1\left(u_{x}\right)^{2}} e^{-\left(2 \pi i k U_{o}\right)(T-s)} .
\end{aligned}
$$

We are now ready to apply the main estimate (29) in Lemma 3. By use of Lemma 3 we get that

$$
\begin{gathered}
\int_{0}^{t-\delta} \pi i k e^{-\left(4 \pi^{2} \nu k^{2}+2 \pi i k U_{o}\right)(t-s)} \hat{u} * \hat{u} d s \\
\leq \frac{C}{\left|k U_{o}\right|} \int_{0}^{t-\delta} \pi|k| \operatorname{esssup}_{\left[s, s+\frac{1}{2 k U_{o}}\right]}\left|\frac{\partial\left(e^{-\left(4 \pi^{2} \nu k^{2}+2 \pi i k U_{o}\right)(t-s)} \hat{u} * \hat{u}\right)}{\partial s}\right| d s .
\end{gathered}
$$


Now

$$
\begin{array}{r}
\frac{\partial\left(e^{-\left(4 \pi^{2} \nu k^{2}+2 \pi i k U_{o}\right)(t-s)} \hat{u} * \hat{u}\right)}{\partial s}=4 \pi^{2} \nu k^{2} e^{-\left(4 \pi^{2} \nu k^{2}+2 \pi i k U_{o}\right)(t-s)} \hat{u} * \hat{u} \\
+2 e^{-\left(4 \pi^{2} \nu k^{2}\right)(t-s)}\left(\hat{u} e^{\left.-2 \pi i k U_{o}\right)(t-s)}\right) * \frac{\partial\left(\hat{u} e^{\left.-2 \pi i k U_{o}\right)(t-s)}\right)}{\partial s} .
\end{array}
$$

By using the Fourier transform of the Navier-Stokes equation (36) we get that

$$
\begin{gathered}
\int_{0}^{t-\delta} \pi i k e^{-\left(4 \pi^{2} \nu k^{2}+2 \pi i k U_{o}\right)(t-s)} \hat{u} * \hat{u} d s \\
\leq \frac{\pi}{U_{o}} \int_{0}^{t-\delta} \operatorname{essipup}_{\left[s, s+\frac{1}{2 k U_{o}}\right]}\left(\nu k^{2}\left|e^{-\left(4 \pi^{2} \nu k^{2}+2 \pi i k U_{o}\right)(t-s)} \hat{u} * \hat{u}\right|\right. \\
\left.+2\left|e^{-\left(4 \pi^{2} \nu k^{2}\right)(t-s)}\left(\hat{u} e^{\left.-2 \pi i k U_{o}\right)(t-s)}\right) * \frac{\partial\left(\hat{u} e^{\left.-2 \pi i k U_{o}\right)(t-s)}\right)}{\partial s}\right|\right) d s \\
\leq \frac{\pi}{U_{o}} \int_{0}^{t-\delta} \operatorname{essipup}\left(4 \pi^{2} \nu k^{2}\left|e^{-\left(4 \pi^{2} \nu k^{2}+2 \pi i k U_{o}\right)(t-s)} \hat{u} * \hat{u}\right|\right. \\
+2 \mid e^{-\left(4 \pi^{2} \nu k^{2}\right)(t-s)} \hat{u} *\left(-4 \pi^{2} \nu k^{2} \hat{u} e^{-\left(2 \pi i k U_{o}\right)(T-s)}\right. \\
\left.\left.-\frac{1}{2} \widehat{\partial_{x} u^{2}} e^{-\left(2 \pi i k U_{o}\right)(T-s)}+\partial_{x}^{-1}\left(u_{x}\right)^{2} e^{-\left(2 \pi i k U_{o}\right)(T-s)}\right) \mid\right) d s .
\end{gathered}
$$

Now we square the last expression above and sum in $k$. Then using the Plancherel identity we get that

$$
\begin{aligned}
& \sum_{k \neq 0}\left|\int_{0}^{t-\delta} \pi i k e^{-\left(4 \pi^{2} \nu k^{2}+2 \pi i k U_{o}\right)(t-s)} \hat{u} * \hat{u} d s\right|^{2} \\
& \leq \sum_{k \neq 0}\left|\frac{12 \pi^{3} \nu}{U_{o}} \int_{0}^{t-\delta} e^{-4 \pi^{2} \nu k^{2}(t-s)}\right| \widehat{u^{2}} \mid(k) d s \\
& \quad+\frac{2 \pi^{2}}{U_{o}} \int_{0}^{t-\delta}|k| e^{-4 \pi^{2} \nu k^{2}(t-s)}\left|\widehat{u^{3}}\right|(k) d s \\
& +\left.\frac{1}{U_{o}} \int_{0}^{t-\delta} \frac{1}{|k|} e^{-4 \pi^{2} \nu k^{2}(t-s)}\left|\widehat{u\left(u_{x}\right)^{2}}\right|(k) d s\right|^{2} \\
& =\frac{C}{U_{o}^{2}} \operatorname{ess~sup}_{[0, t-\delta]}\left(|u|_{4}^{4}+|u|_{\infty}^{2}|u|_{4}^{4}+|u|_{\infty}^{2}\left|u_{x}\right|_{4}^{4}\right) \\
& =\frac{C}{U_{o}^{2}} \operatorname{ess} \sup \left(1+|u|_{\infty}^{2}\right)\left(|u|_{4}^{4}+\left|u_{x}\right|_{4}^{4}\right)
\end{aligned}
$$

where we have used Lemma 4 to estimate the integrals and pulled $u$, in the sup norm, out of the (last two) $L^{2}$ norms in the last step. This finally produces the inequality

$$
\sum_{k \neq 0}\left|\int_{0}^{t-\delta} \pi i k e^{-\left(4 \pi^{2} \nu k^{2}+2 \pi i k U_{o}\right)(t-s)} \hat{u} * \hat{u} d s\right|^{2} \leq \frac{C}{U_{o}^{2}} \operatorname{ess} \sup _{[0, t-\delta]}\left(\left(1+|u|_{\infty}^{2}\right)\left(|u|_{4}^{4}+\left|u_{x}\right|_{4}^{4}\right)\right)
$$


Secondly consider the term

$$
\begin{aligned}
& \sum_{k \neq 0}\left|\int_{0}^{t-\delta} e^{-\left(4 \pi^{2} \nu k^{2}+2 \pi i k U_{o}\right)(t-s)} 2 \partial_{x}^{-1\left(u_{x}\right)^{2}}(k, s) d s\right|^{2} \\
& =\sum_{k \neq 0}\left|\int_{0}^{t-\delta} e^{-\left(4 \pi^{2} \nu k^{2}+2 \pi i k U_{o}\right)(t-s)} \frac{\hat{u}_{x} * \hat{u}_{x}}{\pi i k} d s\right|^{2} .
\end{aligned}
$$

We write

$$
\begin{aligned}
e^{-\left(2 \pi i k U_{o}\right)(T-s)} \hat{u}_{x} * \hat{u}_{x} & =e^{-\left(2 \pi i k U_{o}\right)(T-s)} \sum_{l \neq k, 0} \hat{u}_{x}(k-l) \hat{u}_{x}(l) \\
& =\sum_{l \neq k, 0} e^{-\left(2 \pi i(k-l) U_{o}\right)(T-s)} \hat{u}_{x}(k-l) e^{-\left(2 \pi i l U_{o}\right)(T-s)} \hat{u}_{x}(l) \\
& ={\widehat{u_{x} e}}^{-\left(2 \pi i k U_{o}\right)(T-s)} *{\widehat{u_{x} e}}^{-\left(2 \pi i k U_{o}\right)(T-s)} .
\end{aligned}
$$

Fourier transforming the $x$ derivative of the Navier-Stokes equation and multiplying it with an exponential gives

$$
\begin{array}{r}
\frac{\partial\left(e^{-\left(2 \pi i k U_{o}\right)(T-s)} \hat{u}_{x}\right)}{\partial s}=-4 \pi^{2} \nu k^{2} \hat{u}_{x} e^{-\left(2 \pi i k U_{o}\right)(T-s)} \\
+2 \pi^{2} k^{2} \widehat{u^{2}} e^{-\left(2 \pi i k U_{o}\right)(T-s)}+2 \pi i k \partial_{x}^{\widehat{-1}\left(u_{x}\right)^{2}} e^{-\left(2 \pi i k U_{o}\right)(T-s)}
\end{array}
$$

Then we apply the main estimate (29) in Lemma 3. By use of Lemma 3] we get that

$$
\begin{gathered}
\int_{0}^{t-\delta} e^{-\left(4 \pi^{2} \nu k^{2}+2 \pi i k U_{o}\right)(t-s)} \frac{\hat{u}_{x} * \hat{u}_{x}}{\pi i k} d s \\
\leq \frac{1}{2 U_{o}} \int_{0}^{t-\delta} \frac{1}{\pi k^{2}} \operatorname{essips}_{\left[s, s+\frac{1}{2 k U_{o}}\right]}\left|\frac{\partial\left(e^{-\left(4 \pi^{2} \nu k^{2}+2 \pi i k U_{o}\right)(t-s)} \hat{u}_{x} * \hat{u}_{x}\right.}{\partial s}\right| d s .
\end{gathered}
$$

Now

$$
\begin{array}{r}
\frac{\partial\left(e^{-\left(4 \pi^{2} \nu k^{2}+2 \pi i k U_{o}\right)(t-s)} \hat{u}_{x} * \hat{u}_{x}\right)}{\partial s}=4 \pi^{2} \nu k^{2} e^{-\left(4 \pi^{2} \nu k^{2}+2 \pi i k U_{o}\right)(t-s)} \hat{u}_{x} * \hat{u}_{x} \\
+2 e^{-\left(4 \pi^{2} \nu k^{2}\right)(t-s)}\left(\hat{u}_{x} e^{\left.-2 \pi i k U_{o}\right)(t-s)}\right) * \frac{\partial\left(\hat{u}_{x} e^{-2 \pi i k U_{o}(t-s)}\right)}{\partial s}
\end{array}
$$


By using the Fourier transform of the Navier-Stokes equation (39) we get that

$$
\begin{aligned}
& \int_{0}^{t-\delta} e^{-\left(4 \pi^{2} \nu k^{2}+2 \pi i k U_{o}\right)(t-s)} \frac{\hat{u}_{x} * \hat{u}_{x}}{\pi i k} d s \\
& \leq \frac{1}{2 \pi U_{o}} \int_{0}^{t-\delta} \frac{1}{k^{2}} \operatorname{ess}_{\left[s, s+\frac{1}{2 k U_{o}}\right]}\left(4 \pi^{2} \nu k^{2}\left|e^{-\left(4 \pi^{2} \nu k^{2}+2 \pi i k U_{o}\right)(t-s)} \hat{u}_{x} * \hat{u_{x}}\right|\right. \\
& \left.+2\left|e^{-\left(4 \pi^{2} \nu k^{2}\right)(t-s)}\left(\hat{u}_{x} e^{\left.-2 \pi i k U_{o}\right)(t-s)}\right) * \frac{\partial\left(\hat{u}_{x} e^{\left.-2 \pi i k U_{o}\right)(t-s)}\right)}{\partial s}\right|\right) d s \\
& \leq \frac{1}{2 \pi U_{o}} \int_{0}^{t-\delta} \operatorname{essup}_{\left[s, s+\frac{1}{2 k U_{o}}\right]}\left(4 \pi^{2} \nu\left|e^{-\left(4 \pi^{2} \nu k^{2}+2 \pi i k U_{o}\right)(t-s)} \hat{u}_{x} * \hat{u}_{x}\right|\right. \\
& +2 \mid e^{-\left(4 \pi^{2} \nu k^{2}\right)(t-s)} \hat{u}_{x} *\left(-4 \pi^{2} \nu \hat{u}_{x} e^{-\left(2 \pi i k U_{o}\right)(T-s)}\right. \\
& \left.\left.-2 \pi^{2} \widehat{u^{2}} e^{-\left(2 \pi i k U_{o}\right)(T-s)}-\frac{4 \pi^{2}}{2 \pi i k} \partial_{x}^{-1\left(u_{x}\right)^{2}} e^{-\left(2 \pi i k U_{o}\right)(T-s)}\right) \mid\right) d s .
\end{aligned}
$$

Now we square the last expression above and sum in $k$. Then using the Plancherel identity we get that

$$
\begin{gathered}
\sum_{k \neq 0} \mid \int_{0}^{t-\delta} e^{-\left.\left(4 \pi^{2} \nu k^{2}+2 \pi i k U_{o}\right)(t-s) \frac{\hat{u}_{x} * \hat{u}_{x}}{\pi i k} d s\right|^{2}} \\
\leq \sum_{k \neq 0}\left|\frac{2 \nu \pi}{U_{o}}\right| \int_{0}^{t-\delta} e^{-4 \pi^{2} \nu k^{2}(t-s)}\left|\widehat{\left(u_{x}\right)^{2}}\right|(k) d s \\
\quad+\frac{\pi}{2 U_{o}} \int_{0}^{t-\delta} e^{-4 \pi^{2} \nu k^{2}(t-s)}\left|\widehat{u_{x} u^{2}}\right|(k) d s \\
+\left.\frac{2 \pi}{U_{o}} \int_{0}^{t-\delta} e^{-4 \pi^{2} \nu k^{2}(t-s)}\left|\partial_{x}^{-1}\left[\widehat{u_{x} \partial_{x}^{-1}}\left(u_{x}\right)^{2}\right]\right|(k) d s\right|^{2} \\
=\frac{C}{U_{o}^{2}} \underset{[0, t-\delta]}{\operatorname{ess} \sup }\left(\left|u_{x}\right|_{4}^{4}+|u|_{\infty}^{2}\left(|u|_{4}^{2}\left|u_{x}\right|_{4}^{2}+\left|u_{x}\right|_{4}^{4}\right)\right) \\
=\frac{C}{U_{o}^{2}} \operatorname{ess} \sup \left(1+|u|_{\infty}^{2}\right)\left(|u|_{4}^{2}+\left|u_{x}\right|_{4}^{2}\right)\left|u_{x}\right|_{4}^{2},
\end{gathered}
$$

where we have used Lemma 4 to estimate the integrals, pulled $u$ in the sup norm out of the (last two) $L^{2}$ norms in the last step and integrated by parts to get

$$
\partial_{x}^{-1}\left[u_{x} \partial_{x}^{-1}\left(u_{x}\right)^{2}\right]=\partial_{x}^{-1}\left[u\left(u_{x}\right)^{2}\right] .
$$

This finally produces the inequality

$$
\sum_{k \neq 0}\left|\int_{0}^{t-\delta} e^{-\left(4 \pi^{2} \nu k^{2}+2 \pi i k U_{o}\right)(t-s)} \frac{\hat{u}_{x} * \hat{u}_{x}}{\pi i k} d s\right|^{2} \leq \frac{C}{U_{o}^{2}} \operatorname{ess~sup~}_{[0, t-\delta]}\left(\left(1+|u|_{\infty}^{2}\right)\left(|u|_{4}^{2}+\left|u_{x}\right|_{4}^{2}\right)\left|u_{x}\right|_{4}^{2}\right) .
$$

Combining the estimates (38) and (41) gives the $L^{2}$ estimate

$$
\begin{array}{r}
|u|_{2}^{2} \leq 9 \sum_{k \neq 0} h_{k}\left|A_{t}^{k}\right|^{2}+\frac{C}{\left|U_{o}\right|^{2}} \underset{s \in[0, t]}{\operatorname{esssup}}\left(1+\|u\|_{\frac{5}{4}}^{2}\right)\|u\|_{\frac{5}{4}^{+}}^{4} \\
+\delta^{2} C \underset{s \in[0, t]}{\operatorname{esssup}}\|u\|_{\frac{5}{4}}^{4}
\end{array}
$$


by use of Sobolev's inequality

$$
\left|u_{x}\right|_{4} \leq C\|u\|_{\frac{5}{4}}{ }^{+}
$$

and the Gagliardo-Nirenberg inequalities.

We now act on the integral equation with the operator $\partial_{x}^{(5 / 4)^{+}}$to estimate the derivative $\partial_{x}^{(5 / 4)^{+}} u$ :

$$
\begin{aligned}
\partial_{x}^{(5 / 4)^{+}} u(x, t)= & \sum_{k}\left[(2 \pi i k)^{(5 / 4)^{+}} h_{k}^{1 / 2} A_{t}^{k}\right. \\
& \left.+\frac{1}{2} \int_{0}^{t}(2 \pi i k)^{(5 / 4)^{+}} e^{-\left(4 \pi^{2} \nu k^{2}+2 \pi i k U_{o}\right)(t-s)}\left(2 \widehat{\partial_{x}^{-1}\left(u_{x}\right)^{2}}-\widehat{\partial_{x} u^{2}}\right)(k, s) d s\right] e_{k}(x)
\end{aligned}
$$

where $e_{k}=e^{2 \pi i k x}$ are the Fourier components and the $A_{t}^{k}$ are the Ornstein-Uhlenbeck processes (9).

By the orthogonality of the $e_{k}$ s the $L^{2}$ norm of $u$ is

$$
\begin{aligned}
& \left|\partial_{x}^{(5 / 4)^{+}} u\right|_{2}^{2}=\sum_{k \neq 0}\left[(2 \pi|k|)^{(5 / 2)^{+}} h_{k}\left|A_{t}^{k}\right|^{2}\right. \\
& \left.+\frac{1}{4}\left|\int_{0}^{t}(2 \pi i k)^{(5 / 4)^{+}} e^{-\left(4 \pi^{2} \nu k^{2}+2 \pi i k U_{o}\right)(t-s)}\left(2 \partial_{x}^{-1}\left(u_{x}\right)^{2}-\widehat{\partial_{x} u^{2}}\right)(k, s) d s\right|^{2}\right] .
\end{aligned}
$$

First we estimate the integral from $t-\delta$ to $t$. The $L^{2}$ norm of this integral is

$$
\left.\sum_{k \neq 0} \frac{1}{4} \mid \int_{t-\delta}^{t}(2 \pi i k)^{(5 / 4)^{+}} e^{-\left(4 \pi^{2} \nu k^{2}+2 \pi i k U_{o}\right)(t-s)}\left(2 \widehat{\partial_{x}^{-1}\left(u_{x}\right.}\right)^{2}-\widehat{\partial_{x} u^{2}}\right)\left.(k, s) d s\right|^{2},
$$

and it is estimated by

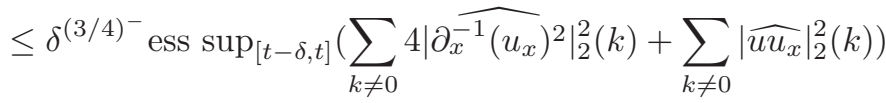

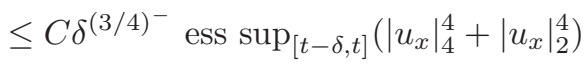

by Plancherel's identity, the Gagliardo-Nirenberg inequalities and Lemma 4.

The integral from 0 to $t-\delta$,

$$
\begin{aligned}
& \left.\sum_{k \neq 0} \frac{1}{4} \mid \int_{0}^{t-\delta}(2 \pi i k)^{(5 / 4)^{+}} e^{-\left(4 \pi^{2} \nu k^{2}+2 \pi i k U_{o}\right)(t-s)}\left(2 \widehat{\partial_{x}^{-1}\left(u_{x}\right.}\right)^{2}-\widehat{\partial_{x} u^{2}}\right)\left.(k, s) d s\right|^{2} \\
& \leq \frac{C}{U_{o}^{2}} \operatorname{ess~sup}_{[0, t-\delta]}\left(\left|u_{x}\right|_{4}^{4}+|u|_{\infty}^{2}\left(|u|_{4}^{2}+\left|u_{x}\right|_{4}^{2}\right)\left|u_{x}\right|_{4}^{2}\right),
\end{aligned}
$$

is estimated by similar computations as above. We need to work a little harder than above to get rid of the $k^{2}$ terms stemming from the Laplacian in the Navier-Stokes equation. Combining the two estimates (45) and (46) we get

$$
\begin{array}{r}
\left|\partial_{x}^{(5 / 4)^{+}} u\right|_{2}^{2} \leq 9 \sum_{k \neq 0}(2 \pi|k|)^{(5 / 2)^{+}} h_{k}\left|A_{t}^{k}\right|^{2}+\frac{C}{\left|U_{o}\right|^{2}} \underset{s \in[0, t]}{\operatorname{esssup}}\left(1+\|u\|_{\frac{5}{4}}^{2}\right)\|u\|_{\frac{5}{4}}^{4} \\
+\delta^{(3 / 4)^{-}} C \underset{s \in[0, t]}{\operatorname{ess} \sup _{s}\|u\|_{\frac{5}{4}}^{4}}
\end{array}
$$

by Sobolev's inequality (43) and the Gagliardo-Nirenberg inequalities. 
Now we combine the inequalities (42) and (47) to get

$$
\begin{array}{r}
\|u\|_{\frac{5}{4}^{+}}^{2} \leq 9 \sum_{k \neq 0}\left(1+(2 \pi|k|)^{(5 / 2)^{+}}\right) h_{k}\left|A_{t}^{k}\right|^{2}+\frac{C}{\left|U_{o}\right|^{2}} \underset{s \in[0, t]}{\operatorname{esssup}\left(1+\|u\|_{\frac{5}{4}}^{2}\right)\|u\|_{\frac{5}{4}^{+}}^{4}} \\
+\delta^{(3 / 4)^{-}} C \underset{s \in[0, t]}{\operatorname{ess} \sup }\|u\|_{\frac{5}{4}}^{4}
\end{array}
$$

and finally adding the term with the initial condition $u^{0}$ to the integral equation (26) we get the estimate

$$
\begin{aligned}
\|u\|_{\frac{5}{4}}^{2} & \leq 16 \sum_{k \neq 0}\left(1+(2 \pi|k|)^{(5 / 2)^{+}}\right) h_{k}\left|A_{t}^{k}\right|^{2}+16\left\|K * u^{0}\right\|_{\frac{5}{4}+}^{2} \\
& +\frac{C}{\left|U_{o}\right|^{2}} \underset{s \in[0, t]}{\operatorname{ess} \sup }\left(1+\|u\|_{\frac{5}{4}}^{2}\right)\|u\|_{\frac{5}{4}+}^{4}+\delta^{(3 / 4)^{-}} C \underset{s \in[0, t]}{\operatorname{ess} \sup }\|u\|_{\frac{5}{4}+}^{4} .
\end{aligned}
$$

Thus by Lemma 5 ,

$$
E\left(\operatorname{ess} \sup \|u\|_{\frac{5}{4}^{+}}^{2}\right) \leq \frac{\left|U_{o}\right|}{\sqrt{C}}
$$

if

$$
16 \sum_{k \neq 0} \frac{\left(1+(2 \pi|k|)^{(5 / 2)^{+}}\right) h_{k}}{8 \pi^{2} k^{2}}+16 E\left(\left\|K * u^{0}\right\|_{\frac{5}{4}}^{2}\right) \leq \frac{2\left|U_{o}\right|}{3 \sqrt{C}}-1
$$

by making $\delta$ arbitrarily small. We set $\frac{1}{\sqrt{C}}$ equal to a new constant $C$ and conclude that (33) imples (34).

Lemma 5. Consider the inequality

$$
x(t)-\left(b x^{3}(t)+c x^{2}(t)\right) \leq a+d, \quad x(0)=x_{0},
$$

where $E(d)=0$ and $b>0, c>0$ are deterministic. If

$$
E(a)<\frac{2}{3}\left(1+\frac{c^{2}}{3 b}\right) x_{\max }-\frac{c}{9 b},
$$

where $x_{\max }=\frac{c}{3 b}\left(\sqrt{\frac{3 b}{c^{2}}+1}-1\right)$ and $E(x(0))<x_{\max }$, then $E(x(t)) \leq x_{\max }$.

Proof. $f(x)=x-b x^{3}-c x^{2}$ obtains its maximum at $x_{\max }$; thus if $E(a)=E(a+d)<$ $\frac{2}{3}\left(1+\frac{c^{2}}{3 b}\right) x_{\max }-\frac{c}{9 b}=f\left(x_{\max }\right)$, then $E(x(t))$ must stay below the value $x_{\max }$ where $f(x)$ obtains its maximum, assuming that $E(x(0))<x_{\max }$.

REMARK 2. Corollary 2 is the resolution of a famous question in turbulence: Is turbulence always caused by the blow-up of the velocity $u$ ? The answer according to Theorem 2 is no; the solutions are not singular. However, they are not smooth either, contrary to the belief, stemming from Leray's theory [20, that if solutions are not singular, then they are smooth. By Corollary 2 the solutions are Hölder continuous with exponent $3 / 4$ in one dimension. This confirms a conjecture made by Onsager [28] in 1945. In particular the gradient $\partial_{x} u$ is not continuous in general.

We can now prove that ess $\sup _{t \in[0, \infty)}\|u(t)\|_{\frac{5}{4}}^{2}$ is bounded with probability one. 
Lemma 6. For all $\epsilon>0$ there exists an $R$ such that

$$
\mathbb{P}\left(\underset{t \in[0, \infty)}{\operatorname{ess} \sup }\|u(t)\|_{\frac{5}{4}}^{2}+<R\right)>1-\epsilon .
$$

Proof. By Chebychev's inequality and the estimate (34) we get that

$$
\mathbb{P}\left(\operatorname{ess~sup}_{t \in[0, \infty)}\|u(t)\|_{\frac{5}{4}^{+}}^{2} \geq R\right)<\frac{C\left|U_{o}\right|}{R}<\epsilon
$$

for $R$ sufficiently large.

4.2. Existence of global turbulent solutions. In this section we prove the existence of the turbulent solutions of the initial value problem (20). The following theorem states the existence of turbulent solutions in one dimension.

THEOREM 2. If

$$
\begin{aligned}
& E\left(\left\|u_{o}\right\|_{\frac{5}{4}}^{2}\right)+E\left(\left\|K * u^{0}\right\|_{\frac{5}{4}}^{2}\right) \\
& =\frac{1}{2} \sum_{k \neq 0} \frac{\left(1+(2 \pi|k|)^{(5 / 2)^{+}}\right)}{(2 \pi|k|)^{2}} h_{k}+E\left(\left\|K * u^{0}\right\|_{\frac{5}{4}}^{2}\right) \\
& \quad \leq \frac{1}{24} C\left|U_{o}\right|-\frac{1}{16},
\end{aligned}
$$

where the uniform flow $U_{o}$ is sufficiently large so that the a priori bound (34) holds, then the integral equation (26) has a unique global solution $u(x, t)$ in the space $C\left([0, \infty) ; \mathcal{L}_{\left(\frac{5}{4}+, 2\right)}^{2}\right), u$ is adapted to the filtration generated by the stochastic process

$$
u_{o}(x, t)=\sum_{k \neq 0} h_{k}^{1 / 2} A_{t}^{k} e_{k}
$$

and

$$
E\left(\int_{0}^{t}\|u\|_{\frac{5}{4}}^{2}+d s\right)<C\left|U_{o}\right| t .
$$

Proof. We let $w=u-v$ and $\alpha=u+v$ where $u$ and $v$ are two solutions of the integral equation. We start by writing

$$
w(x, t)=-\sum_{k \neq 0}\left[\int_{0}^{t} e^{-\left(4 \nu \pi^{2} k^{2}+2 \pi i k U_{o}\right)(t-s)}\left(\widehat{w \cdot \alpha_{x}}+\widehat{\alpha \cdot w_{x}}-\frac{\widehat{\alpha_{x} w_{x}}}{2 \pi i k}\right)(k, s) d s\right] e_{k}(x),
$$

where $e_{k}=e^{2 \pi i k x}$. Then by Lemma 4 ,

$$
\begin{aligned}
& |w|_{2}^{2} \leq \sum_{k \neq 0}\left|\int_{0}^{t} e^{-\left(4 \nu \pi^{2} k^{2}+2 \pi i k U_{o}\right)(t-s)}\left(\widehat{w \alpha_{x}}+\widehat{\alpha w_{x}}-\frac{\widehat{\alpha_{x} w_{x}}}{2 \pi i k}\right)(k, s) d s\right|^{2} \\
& \leq C t^{2} \underset{[0, t]}{\operatorname{ess} \sup }\left(|w|_{\infty}^{2}\left|\alpha_{x}\right|_{2}^{2}+|\alpha|_{\infty}^{2}\left|w_{x}\right|_{2}^{2}+\left|\alpha_{x}\right|_{4}^{4}\left|w_{x}\right|_{4}^{4}\right) \\
& \leq C t^{2} \underset{[0, t]}{\operatorname{ess} \sup }\|\alpha\|_{\frac{5}{4}}^{4}\|w\|_{\frac{5}{4}}^{4}+
\end{aligned}
$$


Similarly

$$
\begin{array}{r}
\left|\partial_{x}^{\frac{5^{+}}{4}} w\right|_{2}^{2} \leq \sum_{k \neq 0}\left|\int_{0}^{t}(2 \pi|k|)^{\frac{5}{4}^{+}} e^{-\left(4 \nu \pi^{2} k^{2}+2 \pi i k U_{o}\right)(t-s)}\left(\widehat{w \alpha_{x}}+\widehat{\alpha w_{x}}\right)(k, s) d s\right|^{2} \\
\leq C t^{\frac{1}{6}{ }^{-}} \underset{[0, t]}{\operatorname{ess} \sup }\|\alpha\|_{\frac{5}{4}}^{4}\|w\|_{\frac{5}{4}^{+}}^{4}
\end{array}
$$

by Lemma 4. Combining those two estimates we get that

$$
\underset{[0, t]}{\operatorname{ess} \sup }\left\|w^{n+1}\right\|_{\frac{5}{4}}^{2} \leq C t^{\frac{1}{6}}{ }_{[0, t]}^{\operatorname{ess} \sup }\left\|\alpha^{n}\right\|_{\frac{5}{4}}^{4}+\underset{[0, t]}{\operatorname{ess} \sup }\left\|w^{n}\right\|_{\frac{5}{4}+}^{4}
$$

for the iteration based on the integral equation (26), and $t$ small. Now the expectation of $\left\|\alpha^{n}\right\|_{\frac{5}{4}}^{4}\left\|w^{n}\right\|_{\frac{5}{4}}^{2}$ can be taken to be bounded by a constant, independent of $n$. This implies that the $\left\|\alpha^{n}\right\|_{\frac{5}{4}}^{4}\left\|w^{n}\right\|_{\frac{5}{4}}^{2}$ norms are bounded with probability one, by an argument similar to that in Lemma 6. Thus

$$
E\left(\underset{[0, t]}{\operatorname{ess} \sup }\left\|w^{n+1}\right\|_{\frac{5}{4}}^{2}\right) \leq C{t^{\frac{1}{6}}}^{-} E\left(\operatorname{ess~sup}_{[0, t]}\left\|w^{n}\right\|_{\frac{5}{4}}^{2}\right),
$$

with probability one, but a constant $C$ that depends on functions in the set

$$
E\left(\left\|\alpha^{n}\right\|_{\frac{5}{4}+}^{4}\left\|w^{n}\right\|_{\frac{5}{4}}^{2}\right) \leq K
$$

in $\mathcal{L}_{\left(\frac{5}{4}+2\right)}^{2}$. By an application of the contraction mapping principle we get that there exists a random variable $\tau$ taking its values almost surely in the interval $(0, t]$, with $t$ small, such that the integral equation (26) defines a contraction on $C\left([0, \tau] ; \mathcal{L}_{\left(\frac{5}{4}+2\right)}^{2}\right)$. This proves the local existence of unique solutions to (26). The global existence uses the bound (34) in Theorem 1, which does not depend on the above set in $\mathcal{L}_{\left(\frac{5}{4}+, 2\right)}^{2}$. Namely, since the norm of $u$ in $\mathcal{L}_{\left(\frac{5}{4}+, 2\right)}^{2}$ is bounded a priori for all $t$, the interval of local existence can be extended to the whole positive $t$-axis $\mathbb{R}^{+}$.

Next we prove an estimate analogous to a Gronwall estimate, which will be used in later sections and also gives an alternative method to prove the uniqueness of the solutions.

Lemma 7. Let $u_{1}$ and $u_{2}$ be two solutions of (26) with initial functions $u_{1}^{o}(x, t)=$ $\sum_{k \neq 0}\left(h_{k}^{1}\right)^{1 / 2} A_{t}^{k} e_{k}$ and $u_{2}^{o}(x, t)=\sum_{k \neq 0}\left(h_{k}^{2}\right)^{1 / 2} A_{t}^{k} e_{k}$, and initial conditions $u_{1}^{0}(x)$ and $u_{2}^{0}(x)$. Then with probability one,

$$
E\left(\left\|u_{1}-u_{2}\right\|_{\frac{5}{4}^{+}}(t)\right) \leq \frac{\left.\left[1-\sqrt{1-4 C\left(t^{\frac{1}{12}}-\right.}+t\right)\left\|w_{0}\right\|_{\frac{5}{4}}+\right)}{2 C\left(t^{\frac{1}{12}}{ }^{-}+t\right)},
$$

where

$$
\begin{aligned}
\left\|w_{0}\right\|_{\frac{5}{4}^{+}} & =E\left(\left\|u_{1}^{0}-u_{2}^{0}\right\|_{\frac{5}{4}^{+}}\right) \\
& +\left[\sum_{k \neq 0} \frac{\left(1+(2 \pi|k|)^{\frac{5^{+}}{2}}\right)}{2(2 \pi k)^{2}}\left|\left(h_{k}^{1}\right)^{1 / 2}-\left(h_{k}^{2}\right)^{1 / 2}\right|^{2}\right]^{\frac{1}{2}} t^{1 / 2} .
\end{aligned}
$$


Proof. We let $u$ and $v$ be two different solutions of the Navier-Stokes equation $w=u-v$ and $\alpha=u+v$ with distinct initial functions $u_{o}$ and $v_{o}$ and write the integral equations for $w$ as

$w(x, t)=w^{0}(x)-\sum_{k \neq 0}\left[\int_{0}^{t} e^{-\left(4 \nu \pi^{2} k^{2}+2 \pi i k U_{o}\right)(t-s)}\left(\widehat{w \cdot \alpha_{x}}+\widehat{\alpha \cdot w_{x}}-\frac{\widehat{\alpha_{x} w_{x}}}{2 \pi i k}\right)(k, s) d s\right] e_{k}(x)$.

The same estimates as in the proof of Theorem 2 give the estimate

$$
\underset{[0, t]}{\operatorname{ess} \sup }\left\|w^{n+1}\right\|_{\frac{5}{4}^{+}} \leq\left\|w_{o}\right\|_{\frac{5}{4}^{+}}+C\left(t^{\frac{1}{12}^{-}}+t\right) \underset{[0, t]}{\operatorname{ess} \sup }\left\|w^{n}\right\|_{\frac{5}{4}}^{2}
$$

with probability one. An iteration of this inequality gives the bound (56).

5. The existence of the invariant measure. In this section we will consider the stochastic Navier-Stokes equation

$$
d w=\left(\nu w_{x x}-w w_{x}+\partial_{x}^{-1}\left(w_{x}\right)^{2}-b\right) d t+\sum_{k \neq 0} h_{k}^{1 / 2} d \beta_{t}^{k} e_{k}
$$

with initial data

$$
w(x, 0)=U_{o}+u^{0}(x),
$$

where $u \in \dot{L}^{2}\left(\mathbb{T}^{1}\right)$, the dot indicating that the mean is equal to zero and $b$ is the constant (21) keeping the mean equal to zero. We will use that the solutions $u(x, t)$, where $w(x, t)=U_{o}+u(x, t)$, exist in $\mathcal{L}_{\left(\frac{5}{4}+, 2\right)}$, by Theorem [2, and that the mean flow $U_{o}$ is constant. Since, by Theorem 2 , we can even take the initial data $u^{0}(x) \in \dot{L}^{2}\left(\mathbb{T}^{1}\right)$, the equation (59) defines a flow on $\dot{L}^{2}\left(\mathbb{T}^{1}\right)$. The reasoning is that if $u^{0}(x) \in \dot{L}^{2}\left(\mathbb{T}^{1}\right)$, then $\left.K * u^{0} \in \dot{W}^{\left(\frac{5}{4}\right.}{ }^{+}, 2\right)\left(\mathbb{T}^{1}\right)$, for $t>0$. This is the physical situation we are interested in, namely fully developed turbulence with nontrivial mean flow, and it applies to most rivers and streams; see [24, 25].

More concretely, we can consider the initial value problem on $\dot{L}^{2}\left(\mathbb{T}^{1}\right)$,

$$
\begin{aligned}
d u= & \left(\nu u_{x x}-U_{o} u_{x}-u u_{x}+\partial_{x}^{-1}\left(u_{x}\right)^{2}-b\right) d t+\sum_{k \neq 0} h_{k}^{1 / 2} d \beta_{t}^{k} e_{k}, \\
& u(x, 0)=u^{0}(x)
\end{aligned}
$$

where

$$
U_{o}=\frac{12}{C} \sum_{k \neq 0} \frac{\left(1+(2 \pi|k|)^{(5 / 2)^{+}}\right)}{(2 \pi|k|)^{2}} h_{k}+\frac{24}{C} E\left(\left\|K * u^{0}\right\|_{\frac{5}{4}}^{2}\right)+\frac{3}{2}+\delta,
$$

$\delta$ being arbitrarily small and $C$ being the constant in Theorem 1 . This stochastic initial value problem is equivalent to the integral equation (26). Then by Theorem 2 the initial value problem (60) defines a flow on $\dot{L}^{2}\left(\mathbb{T}^{1}\right)$.

If $\phi$ is a bounded function on $\dot{L}^{2}\left(\mathbb{T}^{1}\right)$, then the invariant measure $d \mu$ for the SPDE (60) is given by the limit

$$
\lim _{t \rightarrow \infty} E(\phi(u(\omega, t)))=\int_{\dot{L}^{2}\left(\mathbb{T}^{1}\right)} \phi(u) d \mu(u) .
$$


In this section we prove that this limit exists and is unique. We prove below that the limit exists in the $\left.\dot{W}^{\left(\frac{5}{4}\right.}{ }^{+}, 2\right)\left(\mathbb{T}^{1}\right)$ norm, but since these dominate the $\dot{L}^{2}\left(\mathbb{T}^{1}\right)$ norm the conclusions will follow for $\dot{L}^{2}\left(\mathbb{T}^{1}\right)$.

Theorem 3. The integral equation (26) possesses a unique invariant measure.

COROLlary 3. The invariant measure $d \mu$ is ergodic and strongly mixing.

The corollary follows immediately from Doob's Theorem on invariant measures; see for example 30 .

We prove the theorem in three lemmas. First we define a transition probability

$$
P_{t}\left(u^{0}, \Gamma\right)=\mathcal{L}\left(u\left(u^{0}, t\right)\right)(\Gamma), \quad \Gamma \subset \mathcal{E},
$$

where $\mathcal{L}$ is the law of $u(t), u^{0}$ is the initial condition and $\mathcal{E}$ is the natural $\sigma$-algebra of $\dot{L}^{2}\left(\mathbb{T}^{1}\right)$. Then

$$
R_{T}\left(u^{0}, \cdot\right)=\frac{1}{T} \int_{0}^{T} P_{t}\left(u^{0}, \cdot\right) d t
$$

is a probability measure on $\dot{L}^{2}\left(\mathbb{T}^{1}\right)$. By the Krylov-Bogoliubov theorem, see 30, if the sequence of measures $R_{T}$ is tight, then the invariant measure $d \mu$ is the weak limit

$$
d \mu(\cdot)=\lim _{T \rightarrow \infty} \frac{1}{T} \int_{0}^{T} P_{t}\left(u^{0}, \cdot\right) d t .
$$

Namely,

and

$$
R_{T}^{*} d \nu(\Gamma)=\int_{\dot{L}^{2}\left(\mathbb{T}^{1}\right)} R_{T}\left(u^{0}, \Gamma\right) d \nu\left(u^{0}\right)
$$

$$
\left\langle R_{T}^{*} \nu, \phi\right\rangle=\int_{\dot{L}^{2}\left(\mathbb{T}^{1}\right)} \phi\left(u^{0}\right) R_{T}\left(u^{0}, \Gamma\right) d \nu\left(u^{0}\right) \rightarrow \int_{\dot{L}^{2}\left(\mathbb{T}^{1}\right)} \phi\left(u^{0}\right) d \mu\left(u^{0}\right)
$$

as $T \rightarrow \infty$.

LEMMA 8. The sequence of measures

$$
\frac{1}{T} \int_{0}^{T} P_{t}\left(u^{0}, \cdot\right) d t
$$

is tight.

Proof. By the inequality (34),

$$
\frac{1}{T} \int_{0}^{T} E\left(\|u\|_{\frac{5}{4}^{+}}^{2}\right)(t) d t \leq C U_{o} .
$$

The space $\left.\dot{H}^{\frac{5}{4}}\left(\mathbb{T}^{1}\right)=\dot{W}^{\left(\frac{5}{4}\right.}{ }^{+}, 2\right)$ is relatively compact in $\dot{L}^{2}\left(\mathbb{T}^{1}\right)$, so it suffices to show that $u(t)$ lies in a bounded set in $\dot{H}^{\frac{5}{4}}{ }^{+}\left(\mathbb{T}^{1}\right)$ almost surely, or for all $\epsilon>0$, there exists an $R$ such that

$$
\frac{1}{T} \int_{0}^{T} \mathbb{P}\left(\|u(t)\|_{\frac{5}{4}+}^{2}<R\right) d t>1-\epsilon
$$

for $T \geq 1$. But this follows from Chebychev's inequality, similarly as in Lemma 6 namely,

$$
\frac{1}{T} \int_{0}^{T} \mathbb{P}\left(\|u(t)\|_{\frac{5}{4}}+\geq R\right) d t \leq \frac{1}{R} C U_{o}<\epsilon
$$


for $R$ sufficiently large. By Theorem 2 we can take the initial data in $\dot{L}^{2}\left(\mathbb{T}^{1}\right)$. This proves that the sequence of measures is tight.

Next we prove the strong Feller property; see 30 .

Lemma 9. The Markovian semigroup $P_{t}$ generated by the integral equation (26) is strongly Feller.

Proof. We compare the operators

$$
S=\frac{\nu}{2} \partial_{x}^{2}
$$

and

$$
A=-2 \partial_{x}^{-1} u_{x} \partial_{x}
$$

on $\dot{L}^{2}\left(T^{1}\right)$, the $L^{2}$ space of functions with mean zero. The operator $A$ is $S$ bounded, see Kato [18,

$$
\|A v\| \leq a\|v\|+b\|S v\|
$$

with the coefficients $a=0$ and $b=\frac{4}{\nu \sqrt{\lambda_{1}}}\left|u_{x}\right|_{2}$, we have

$$
\|A v\|^{2}=4\left\|\partial_{x}^{-1} u_{x} v_{x}\right\|^{2} \leq 4\left|u_{x}\right|_{2}^{2}\left|v_{x}\right|_{2}^{2} \leq \frac{16}{\nu^{2} \lambda_{1}}\left|u_{x}\right|_{2}^{2}\left|\frac{\nu}{2} \partial_{x}^{2} v\right|_{2}^{2}=\frac{16}{\nu^{2} \lambda_{1}}\left|u_{x}\right|_{2}^{2}\|S v\|^{2}
$$

by Poincaré's inequality, since $v_{x}=0$ has mean zero, where $\lambda_{1}=4 \pi^{2}$. Taking the expectation we get that

$$
E\left(\|A v\|^{2}\right)=\frac{16}{\nu^{2} \lambda_{1}} E\left(\left|u_{x}\right|_{2}^{2}\right)\|S v\|^{2}
$$

since $S$ is deterministic. Now $S$ generates a contraction semi-group and that $A$ is $S$ bounded implies that $S+A$ also generates a contraction semi-group; see Theorem 2.4, page 499 in Kato [18. Moreover, it will be shown by a direct computation below that the operator

$$
B=\frac{\nu}{2} \partial_{x}^{2}-\left(U_{o}+u\right) \partial_{x}-u_{x}
$$

also generates a contraction semi-group, and thus by Theorem 2.7, page 501 in Kato [18, the operator

$$
T=B+S+A
$$

generates a contraction semi-group that we will denote

$$
V(t)=e^{T t} .
$$

This semi-group satisfies the estimate, Kato [18,

$$
\|V(t)\| \leq 1,
$$

and the first spatial derivative of $V$ is also bounded by a constant $M$.

We will now solve the equation

$$
v_{t}=B v
$$

where $B$ is the operator above. The initial value problem

$$
\begin{array}{r}
w_{t}=\nu w_{x x}-\left(U_{o}+u\right) w_{x}-w u_{x}, \\
w(0)=\delta(x)
\end{array}
$$


for the functional derivative $w(x, t)=\frac{\partial u(x, t)}{\partial u(y, 0)}$ is solved by a combination of FeynmannKac and Cameron-Martin; see McKean [23. Namely, after a reversal of time $t-s \rightarrow s$, $w$ can be written as

$$
w\left(x=x_{t}, t\right)=\left.e^{\int_{0}^{t}\left(U_{o}+u\left(s, x_{s}\right)\right) \cdot d x_{s}-\frac{1}{2} \int_{0}^{t}\left|U_{o}+u\left(s, x_{s}\right)\right|^{2} d s} K(x, t)\right|_{x=x_{t}}
$$

where $K(x, t)$ is the periodic heat kernel in one dimension. Thus following McKean [23],

$$
\phi_{t}(u)-\phi_{t}(v)=\int_{\mathbb{T}^{1}} \int_{\mathbb{T}^{1}} B M \int_{0}^{1} \nabla \phi(h) \cdot(u-v)(y, t) w\left(x=x_{t}, t\right) d r d x d y,
$$

where $h=v+(u-v) r$ and $\phi_{t}(u)=E(\phi(u(t))$. BM denotes the Brownian mean over the individual motions. Thus by Lemma 10 and the Schwarz inequality,

$$
\left|\phi_{t}(u)-\phi_{t}(v)\right| \leq 2\left(\lim _{t \rightarrow \infty} \frac{\left|x_{t}\right|}{t}+\left|U_{o}\right|+|u|_{\infty}\right)\|\phi\|_{\infty}|u-v|_{2},
$$

where $x_{t} \in \mathbb{T}^{1}$, since

$$
B M\left(e^{\int_{0}^{t}\left(U_{o}+u\left(s, x_{s}\right)\right) \cdot d x_{s}-\frac{1}{2} \int_{0}^{t}\left|U_{o}+u\left(s, x_{s}\right)\right|^{2} d s}\right)=E\left(e^{\int_{0}^{t}\left(U_{o}+u\left(s, x_{s}\right)\right) \cdot d x_{s}-\frac{1}{2} \int_{0}^{t}\left|U_{o}+u\left(s, x_{s}\right)\right|^{2} d s}\right)=1
$$

or

$$
\begin{aligned}
\left|\phi_{t}(u)-\phi_{t}(v)\right| & \leq C\left(\left|U_{o}\right|+\|u\|_{\frac{5}{4}^{+}}\right)|\phi|_{\infty}|(u-v)|_{2} \\
& \leq C\left(\left|U_{o}\right|+\|u\|_{\frac{5}{4}}+\right)|\phi|_{\infty}\|(u-v)\|_{\frac{5}{4}}{ }^{+}
\end{aligned}
$$

by the Gagliardo-Nirenberg inequalities. This implies that the Markovian semi-group $P_{t}$ is strongly Feller, both in $\dot{L}^{2}$ and in $\dot{W}^{\left(\frac{5}{4}^{+}, 2\right)}$, since $\|u\|_{\frac{5}{4}}+$ is bounded, with probability one, by Lemma 6 .

Now repeating this argument with the operator

$$
T=\nu \partial_{x}^{2}+2 \partial_{x}^{-1} u_{x} \partial_{x}-\left(U_{o}+u\right) \partial_{x}-u_{x}
$$

instead of $B$, we get the inequality

$$
\phi_{t}(u)-\phi_{t}(w)=\int_{0}^{1} \int_{0}^{1} T M \int_{0}^{1} \nabla \phi(h) \cdot(u-w)(y, t) v(x, t) d r d x d y,
$$

where $T M$ denotes the mean over the densities of the semi-group $V(t)$ generated by $T$. We do not know these densities explicitly as those above but we can still estimate them and their derivatives with respect to $x$ using the estimates (62) on $V(t)$ above. By estimates similar to the above and integration by parts combined with Itô's lemma we get the estimate

$$
\left|\phi_{t}(u)-\phi_{t}(w)\right| \leq C|\phi|_{\infty}\|u-w\|
$$

either in $\dot{L}^{2}$ or $\dot{W}^{\left(\frac{5}{4}+, 2\right)}$. This proves the strong Feller property.

LEMMA 10.

$$
\begin{gathered}
\int_{\mathbb{T}^{1}} \nabla \phi(h) \cdot(u-v)(y, t) w\left(x=x_{t}, t\right) d x \\
=\int_{\mathbb{T}^{1}}\left(\frac{x_{t}}{t}+U_{o}+u\left(t, x_{t}\right)\right) \phi(h) \cdot(u-v)(y, t) w\left(x=x_{t}, t\right) d x .
\end{gathered}
$$


Proof. Extending $u$ and $w$ to all of $\mathbb{R}$ by use of periodicity we can write

$$
\begin{aligned}
& \int_{\mathbb{T}^{1}} \nabla \phi(h) \cdot(u-v)(y, t) w\left(x=x_{t}, t\right) d x \\
= & \left.\int_{\mathbb{R}} \nabla \phi(h) \cdot(u-v)(y, t) e^{\int_{0}^{t}\left(U_{o}+u\left(s, x_{s}\right)\right) \cdot d x_{s}-\frac{1}{2} \int_{0}^{t}\left|U_{o}+u\left(s, x_{s}\right)\right|^{2} d s} \frac{e^{-\frac{x^{2}}{2 \nu t}}}{(2 \pi \nu t)^{\frac{1}{2}}}\right|_{x=x_{t}} d x \\
= & \int_{\mathbb{R}}\left(\frac{x_{t}}{t}+U_{o}+u\left(t, x_{t}\right)\right) \phi(h)(u-v)(y, t) \\
& \times\left. e^{\int_{0}^{t}\left(U_{o}+u\left(s, x_{s}\right)\right) \cdot d x_{s}-\frac{1}{2} \int_{0}^{t}\left|U_{o}+u\left(s, x_{s}\right)\right|^{2} d s} \frac{e^{-\frac{x^{2}}{2 \nu t}}}{(2 \pi \nu t)^{\frac{1}{2}}}\right|_{x=x_{t}} d x \\
= & \int_{\mathbb{T}^{1}}\left(\frac{x_{t}}{t}+U_{o}+u\left(t, x_{t}\right)\right) \phi(h) \cdot(u-v)(y, t) w\left(x=x_{t}, t\right) d x
\end{aligned}
$$

by integration by parts and Itô's formula, and folding back to $\mathbb{T}^{1}$ again by use of periodicity.

Finally we prove irreducibility, see [30, of $P_{t}$. The proof of this lemma is an application of stochastic control theory.

Lemma 11. The Markovian semigroup $P_{t}$ generated by the integral equation (26) is irreducible.

Proof. We first consider the linear deterministic equation

$$
\begin{aligned}
& z_{t}+U_{o} z_{x}=\nu z_{x x}+w(x, t), \\
& z(x, 0)=0, \quad z(x, T)=b(x)
\end{aligned}
$$

and the deterministic equation

$$
\begin{array}{r}
y_{t}+U_{o} y_{x}=\nu y_{x x}-y y_{x}+\partial_{x}^{-1}\left(y_{x}\right)^{2}-b+Q h(x, t), \\
y(x, 0)=0, \quad y(x, T)=b(x),
\end{array}
$$

where $\left.Q: \dot{H}^{-1} \rightarrow \dot{W}^{\left(\frac{5}{4}\right.}{ }^{+}, 2\right)$, both spaces have mean zero, $b$ keeps the mean equal to zero as in (21) and the kernel $Q$ is empty. We will define the operator $Q$ by coefficients in the sum $\sum_{k \neq 0} h_{k}^{1 / 2} A_{t}^{k} e_{k}$, where the $A_{t}^{k}$ are the Ornstein-Uhlenbeck processes from (9). Then it is easy to check that if $Q f=\sum_{k \neq 0} h_{k}^{1 / 2} f_{k} e_{k}=0$, then $f=0$ since $h_{k}^{1 / 2} \neq 0$ for all $k \neq 0$.

We can pick a function $w \in C\left([0, T] ; \dot{W}^{\left(\frac{5}{4}+, 2\right)}\right)$ such that $z(x, T)=b(x)$ and a corresponding function $h \in L^{2}\left([0, T] ; \dot{H}^{-1}\left(\mathbb{T}^{1}\right)\right)$. Namely, $Q h=z z_{x}-\partial_{x}^{-1}\left(z_{x}\right)^{2}+w$, since the kernel of $Q$ is empty; then $y=z$ is a solution of the deterministic Navier-Stokes equation (66) above. This means that (66) is exactly controllable; see Curtain and Zwart [7].

Now we compare $y$ and the solution $u$ of the integral eqution (26). By the same estimate as in Lemma 7 , we get the inequality

$$
E\left(\|u-y\|_{\frac{5}{4}}\right) \leq E\left(\left\|u_{o}(x, t)-y_{o}(x, t)\right\|_{\frac{5}{4}^{+}}\right) t^{\frac{1}{2}}
$$


as long as $w_{0}=u_{o}(x, t)-y_{o}(x, t)$ is sufficiently small. Here $y$ satisfies the integral equation

$$
y=y_{o}+\int_{0}^{t} K *\left(-y y_{x}+\partial_{x}^{-1}\left(y_{x}\right)^{2}-b\right) d s,
$$

where $K$ is the oscillatory heat kernal in (7) and $y_{o}=\int_{0}^{t} K * w d s$. Now the inequality (56) implies that for $t \leq T$ and $\delta<1$,

$$
E\left(\|u-y\|_{\frac{5}{4}^{+}}\right) \leq E\left(\left\|u_{o}(x, t)-y_{o}(x, t)\right\|_{\frac{5}{4}^{+}}\right) \sqrt{T}<\frac{\epsilon \delta}{2}
$$

since we can pick $h$ such that

$$
E\left(\left\|u_{o}(x, t)-y_{o}(x, t)\right\|_{\frac{5}{4}}\right) \leq \frac{\epsilon \delta}{2 \sqrt{T}}
$$

for $t \leq T$. We are using here that the support of the law of $u_{o}$ is the same in $\dot{W}^{\left(\frac{5}{4}+, 2\right)}\left(\mathbb{T}^{1}\right)$ as in $\dot{L}^{2}\left(\mathbb{T}^{1}\right)$, since the former space is smoothly embedded in the latter, see 29]; and that the closure of the image of $Q$, in the $C^{0}$ (sup in $t$ ) norm, is dense in the support of the law of $u_{o}$; compare Theorems 7.4.1 and 7.4.2 in [30]. This implies that the probability

$$
\begin{aligned}
& \mathbb{P}\left(\|u(T)-b\|_{\frac{5}{4}^{+}} \leq \epsilon\right) \quad \geq \\
& \mathbb{P}\left(\|u(T)-y(T)\|_{\frac{5}{4}}+\leq \frac{\epsilon}{2} \quad \text { and } \quad\|y(T)-b(T)\|_{\frac{5}{4}}+\leq \frac{\epsilon}{2}\right) \geq 1-\delta>0
\end{aligned}
$$

by (67) and Chebychev's inequality, since (66) is exactly controllable.

6. Hack's law. We now use the invariant measure in Section 5 to derive the scaling of the second structure function of the turbulent flow that solves Equation (26).

Lemma 12. The second structure function of the turbulent flow that solves the integral equation (26) scales as

$$
s_{2}(x)=\int_{L^{2}\left(T^{1}\right)}|u(y+x)-u(y)|^{2} d \mu(u) \leq C|x|^{\frac{3}{2}^{+}} .
$$

Proof. By Corollary 2 the solutions of (26) are Hölder continuous with exponent 3/4. Thus

$$
|u(y+x)-u(y)| \leq C\|u\|_{\left(\frac{5}{4}+, 2\right)^{+}|x|^{\frac{3}{4}}} .
$$

Moreover, by Theorem $2 u$ satisfies the bound

$$
E\left(\|u\|_{\left(\frac{5}{4}+, 2\right)}^{2}\right) \leq C\left|U_{o}\right|
$$

by Equation (34). Substituting these bounds into the integral and using that

$$
E\left(\|u\|_{\left(\frac{5}{4}+, 2\right)}^{2}\right)=\int_{L^{2}}\|u\|_{\left(\frac{5}{4}+, 2\right)}^{2} d \mu
$$

gives the estimate. 
The scaling of the structure functions depends on the dimensions, see 14, in three dimensions $s_{2}(x) \sim x^{\frac{2}{3}}$ corresponding to Kolmogorov's scaling.

Hack's law describes how the length $l$ of a main river in a river basin scales with the area $A$ of the basin, see Hack [16],

$$
l=c A^{0.568}
$$

where $c$ is a constant that varies from basin to basin; see Gray [15. Hack's law describes the fact that most river basins have an oval shape; that is, they are longer along the direction of the main river than perpendicular to it. As discussed in the introduction there are several ranges in Hack's law depending on the size of the river basin, but we restrict our discussion here to basins which are large, say $>200 \mathrm{~km}^{2}$, but not too large, say $<25,000 \mathrm{~km}^{2}$; see Mueller [26.

Now Hack's law is proven in the following manner. In [4] the equations describing the sediment flow are linearized about convex (concave in the terminology of geomorphology) surface profiles describing mature surfaces. Then the colored noise generated by the turbulent flow drives the linearized equations and the solutions obtain the same color (scaling); see Theorem 5.3 in [4. The resulting variogram (second structure function) of the surfaces scales with the roughness exponent $\chi=\frac{3}{4}$; see Theorem 5.4 in [4. This determines the roughness coefficient $\chi$ of mature landsurfaces. This roughness applies to the transport limited landsurfaces studied in 4 and is in excellent agreement with numerical simulations [6] and empirical results from Digital Elevation Models [35, of areas where the transport limited assumption applies.

Hack's law is a universal statistical law and applies to detachment limited landsurfaces as well as transport limited ones. In the former case we have to wait for the rock to weather before it can be eroded, whereas in the latter case all the sediment can be eroded if sufficient water is available. There are good reasons to believe that the theory developed in [4] will apply to the detachment limited situation as well but this remains to be shown. However, a question still remained, namely how the spatial roughness $\chi=3 / 4$ of a channel or a riverbed, caused by the turbulent flow eroding the bed, is transported to the whole surface? This question is answered in the paper [5], which studies the meanderings of an experimental stream on an acrylic plate. It is shown in [5] that the meanderings of this experimental stream are caused by noise in the water flow that gives rise to turbulence, and the meandering coefficient of the experimental stream is exactly the roughness coefficient of the turbulent flow or $\chi=3 / 4$. Since the meanders of the experimental stream are expected to reflect exactly the roughness of the flow, this constitutes the first direct verification of the theory presented in this paper. Moreover, the question posed above is answered: The roughness of the meanderings implies that the scaling of the area covered by them is in accordance with Hack's law; see the argument below. This indicates that the river meanderings cover the whole river basin over time, thus endowing the surface of the whole basin with the same spatial roughness as the river channel.

The final step in the following derivation of Hack's law is copied from [6]. 
6.1. The origin of Hack's law. The preceding results allow us to derive some of the fundamental scaling results that are known to characterize fluvial landsurfaces. In particular, the avalanche dimension computed in [6] and derived in [4, given the roughness coefficient $\chi$, allows us to derive Hack's law relating the length of a river $l$ to the area $A$ of the basin that it drains. This is the area of the river network that is given by the avalanche dimensions

$$
A \sim l^{D}
$$

and the avalanche dimension is $D=1+\chi$. This relation says that if the length of the main river is $l$, then the width of the basin in the direction perpendicular to the main river is $l^{\chi}$. Notice that if $L_{\|} \sim L$ is the diameter of the basin along the main river and $L_{\perp} \sim L^{\chi}$ is the width of the basin perpendicular to this direction, then the relation above simply says that the area of the basin is proportional to their product

$$
A \sim L_{\|} L_{\perp}=L^{1+\chi} .
$$

However, this does not take into account the sinuosity of the river

$$
l \sim L_{\|}^{\alpha},
$$

where $\alpha$ is the meandering exponent or sinuosity (fractal dimension) of real rivers. Taking sinuosity into account gives

$$
A \sim L^{\frac{D}{\alpha}}=L^{\frac{1+\chi}{\alpha}}
$$

by substitution into Hack's law (68). It turns out that for real rivers, $\alpha \approx 1.1[22$ is small, so it does not make much difference whether $l$ or $L$ is used in Hack's law. The origin of the statistical law for sinuosity

$$
l \sim L_{\|}^{\alpha}
$$

of real rivers is still a mystery. It is clear from [5] that turbulent flow in the river contributes, but it does not provide the complete explanation for the meandering exponent $\alpha$, as it does for the roughness exponent $\chi$ and Hack's law (68).

Stable scalings for the surface emerge together with the emergence of the separable solutions describing the mature surfaces; see [ $\left[\underline{6}\right.$ and $\left[4\right.$. We note that in this case, $\chi=\frac{3}{4}$ as shown in Lemma 12 above; hence we obtain

$$
\begin{aligned}
l & \sim A^{\frac{1}{1+\chi}} \\
& \approx A^{0.571},
\end{aligned}
$$

a number that is in excellent agreement with observed values of the exponent of Hack's law of 0.568 ; see [15].

Acknowledgments. This research for this paper was started during the author's sabbatical at the University of Granada, Spain. It was also finished in Granada two years later. The author was supported by grant number DMS-0352563 from the National Science Foundation whose support is gratefully acknowledged. Some simulations are being done on a cluster of workstations, funded by a National Science Foundation SCREMS grant number DMS-0112388. The author wishes to thank Professor Juan Soler of the Applied Mathematics Department at the University of Granada for his support, 
and the whole research group, Delia Jiroveanu, José Luis López, Juanjo Nieto, Oscar Sánchez and María José Cáceres for their help and inspiration. His special thanks go to Professor José Martínez Aroza who cheerfully shared his office and the wonders of the fractal universe.

The paper was finished at the Mittag-Leffler Institute in Stockholm, Sweden, whose support is gratefully acknowledged, and also acknowledged is support from the UC Santa Barbara Academic Senate.

\section{REFERENCES}

[1] R. Betchov and W. O. Criminale, Stability of parallel flows, Academic Press, New York, 1967.

[2] B. Birnir, Turbulence of uniform flow, Proceedings of the conference Probability, Geometry and Integrable Systems, MSRI Dec. 2005, MSRI Publication Series nr. 55, Cambridge University Press (2008), Available at: http://www.math.ucsb.edu/ birnir/papers.

[3] B. Birnir, Uniqueness, an invariant measure and Kolmogorov's scaling for the stochastic NavierStokes equation, Preprint (2007), Available at: http://www.math.ucsb.edu/ ${ }^{\sim}$ birnir/papers.

[4] B. Birnir, J. Hernández, and T. R. Smith, The stochastic theory of fluvial landsurfaces, J. Nonlinear Science 17 (2007), 13-57, DOI:10.1007/s00332-005-0688-3. MR2281138 (2007k:86002)

[5] B. Birnir, Keith Mertens, Vakhtang Putkaradze, and Peter Vorobieff, Meandering of fluid streams on acrylic surface driven by external noise, To appear in Journ. Fluid Mech. (2008).

[6] B. Birnir, T.R. Smith, and G. Merchant, The Scaling of Fluvial Landscapes, Computers and Geoscience 27 (2001), 1189-1216.

[7] R. F. Curtain and H. Zwart, An introduction to infinite-dimensional linear systems theory, Springer, New York, 1995. MR.1351248 (96i:93001)

[8] P. S. Dodds and D. Rothman, Geometry of river networks. I. Scaling, fluctuations and deviations, Phys. Rev. E 63 (2000), 016115.

[9] _ Geometry of river networks. II. Distributions of component size and number, Phys. Rev. E 63 (2000), 016116.

[10] _ Geometry of river networks. III. Characterization of component connectivity, Phys. Rev. E 63 (2000), 016117.

[11] Scaling, universality and geomorphology, Annu. Rev. Earth Planet. Sci. 28 (2000), 571-610.

[12] _ Unified view of scaling laws for river networks, Phys. Rev. E 59 (2000), no. 5, 4865.

[13] S. F. Edwards and D. R. Wilkinson, The surface statistics of granular aggregate, Proc. Roy. Soc. London. Series A (1982). MR661715 (83m:82032)

[14] U. Frisch, Turbulence, Cambridge Univ. Press, Cambridge, 1995. MR.1428905 (98e:76002)

[15] D.M. Gray, Interrelationships of watershed characteristics, Journal of Geophysics Research 66 (1961), no. 4, 1215-1223.

[16] J. Hack, Studies of longitudinal stream profiles in Virginia and Maryland, U.S. Geological Survey Professional Paper 294-B (1957).

[17] R. E. Horton, Erosional development of streams and their drainage basins: A hydrophysical approach to quantitative morphology, Geol. Soc. Am. Bull. 56 (1945), 275-370.

[18] T. Kato, Perturbation theory for linear operators, Springer, New York, 1976. MR0407617|(53:11389)

[19] S. L. Dingman, Fluvial hydrology, W. H. Freeman and Company, New York, 1984.

[20] J. Leray, Sur le mouvement d'un liquide visqueux emplissant l'espace, Acta Math. 63 (1934), no. 3, 193-248. MR 1555394

[21] E. Levi, The science of water, ASCE Press, New York, 1995.

[22] A. Maritan, A. Rinaldo, R. Rigon, A. Giacometti, and I. Rodriguez-Iturbe, Scaling laws for river networks, Phys. Rev. E 53 (1996), 1510.

[23] H. P. McKean, Turbulence without pressure: Existence of the invariant measure, Methods and Applications of Analysis 9 (2002), no. 3, 463-468. MR2032342 (2005d:76023)

[24] A. S. Momin and A. M. Yaglom, Statistical fluid mechanics, vol. 1, MIT Press, Cambridge, MA, 1971.

[25] _ Statistical fluid mechanics, vol. 2, MIT Press, Cambridge, MA, 1975.

[26] J. E. Mueller, Re-evaluation of the relationship of master streams and drainage basins: Reply, Geo. Soc. Amer. Bull. 84 (1973), 3127-3130. 
[27] B. Oksendal, Stochastic differential equations, Springer, New York, 1998. MR.1619188 (99c:60119)

[28] L. Onsager, Statistical hydrodynamics, Nuovo Cimento. 6 (1945), no. 2, 279-287. MR0036116 (12:60f)

[29] G. Da Prato and J. Zabczyk, Stochastic equations in infinite dimensions, Cambridge University Press, Cambridge UK, 1992. MR 1207136 (95g:60073)

[30] — Ergodicity for infinite dimensional systems, Cambridge University Press, Cambridge UK, 1996. MR 1417491 (97k:60165)

[31] I. Rodriguez-Iturbe and A. Rinaldo, Fractal river basins: Chance and self-organization, Cambridge University Press, Cambridge UK, 1997.

[32] T.R. Smith, G.E. Merchant, and B. Birnir, Transient attractors: Towards a theory of the graded stream for alluvial and bedrock channels, Computers and Geosciences 26 (2000), no. 5, 531-541.

[33] T.R. Smith, B. Birnir, and G.E. Merchant, Towards an elementary theory of drainage basin evolution: I. The theoretical basis, Computers and Geoscience 23 (1997), no. 8, 811-822.

[34] T.R. Smith, G.E. Merchant, and B. Birnir, Towards an elementary theory of drainage basin evolution: II. A computational evaluation, Computers and Geoscience 23 (1997), no. 8, 823-849.

[35] J.K. Weissel, L.F. Pratson, and A. Malinverno, The length-scaling of topography, Journal of Geophysical Research 99 (1994), 13997-14012.

[36] E. Welsh, B. Birnir, and A. Bertozzi, Shocks in the evolution of an eroding channel, AMRX Appl. Math. Research eXpress 2006 (2007), 1-27. MR2278493 (2007h:86014) 